JOURNAL OF SYNCHROTRON RADIATION

ISSN 1600-5775

Received 26 May 2015

Accepted 21 September 2015

Edited by A. Momose, Tohoku University, Japan

Keywords: X-rays; phase contrast; computed tomography; mammography.

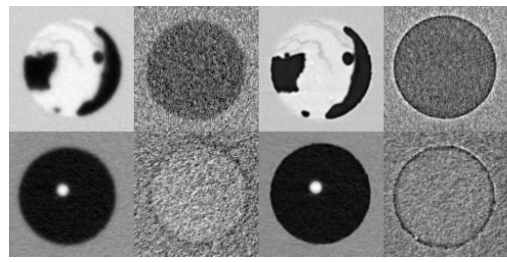

(C) 2015 International Union of Crystallography

\section{A feasibility study of X-ray phase-contrast mammographic tomography at the Imaging and Medical beamline of the Australian Synchrotron}

\author{
Yakov I. Nesterets, ${ }^{\mathrm{a}, \mathrm{b} *}$ Timur E. Gureyev, ${ }^{\mathrm{a}, \mathrm{b}, \mathrm{c}, \mathrm{d}}$ Sheridan C. Mayo, \\ Andrew W. Stevenson, ${ }^{a, e}$ Darren Thompson, ${ }^{a, b}$ Jeremy M. C. Brown, ${ }^{d}$ \\ Marcus J. Kitchen, ${ }^{d}$ Konstantin M. Pavlov, ${ }^{b, d}$ Darren Lockie, ${ }^{f}$ \\ Francesco Brun $^{\mathrm{g}, \mathrm{h}}$ and Giuliana Tromba ${ }^{\mathrm{h}}$
}

${ }^{\mathbf{a} C}$ Commonwealth Scientific and Industrial Research Organisation, Melbourne, Australia, ${ }^{\mathbf{b}}$ School of Science and Technology, University of New England, Armidale, Australia, ${ }^{\mathbf{c}}$ ARC Centre of Excellence in Advanced Molecular Imaging, School of Physics, The University of Melbourne, Parkville, Australia, ${ }^{\mathbf{d}}$ School of Physics and Astronomy, Monash University, Melbourne, Australia, ${ }^{\mathbf{e}}$ Australian Synchrotron, Melbourne, Australia, ${ }^{\mathbf{f}}$ Maroondah BreastScreen, Melbourne, Australia, ${ }^{\mathbf{g}}$ Department of Engineering and Architecture, University of Trieste, Trieste, Italy, and ${ }^{\mathbf{h}}$ Elettra - Sincrotrone Trieste SCpA, Basovizza (Trieste), Italy. *Correspondence e-mail: yakov.nesterets@csiro.au

Results are presented of a recent experiment at the Imaging and Medical beamline of the Australian Synchrotron intended to contribute to the implementation of low-dose high-sensitivity three-dimensional mammographic phase-contrast imaging, initially at synchrotrons and subsequently in hospitals and medical imaging clinics. The effect of such imaging parameters as X-ray energy, source size, detector resolution, sample-to-detector distance, scanning and data processing strategies in the case of propagation-based phase-contrast computed tomography (CT) have been tested, quantified, evaluated and optimized using a plastic phantom simulating relevant breast-tissue characteristics. Analysis of the data collected using a Hamamatsu CMOS Flat Panel Sensor, with a pixel size of $100 \mu \mathrm{m}$, revealed the presence of propagation-based phase contrast and demonstrated significant improvement of the quality of phase-contrast CT imaging compared with conventional (absorption-based) CT, at medically acceptable radiation doses.

\section{Introduction}

Breast cancer is one of the two leading causes of cancer fatalities among women in most industrialized countries. This type of cancer can be very aggressive, with success of the treatment depending heavily on early detection which is currently the most important factor for reducing the morbidity and mortality of the patients. Therefore, health authorities in most countries recommend regular screening of women over 40 years of age, with two-dimensional (2D) X-ray mammography being the main screening and diagnostic technique used for this purpose at present.

Despite the intensive studies of the optimization of conventional (Johns \& Yaffe, 1985) and phase-contrast (Zysk et al., 2012) mammographic imaging systems, the main known problem with this technique is that it produces a relatively high percentage of both false-positive and false-negative results (Pisano et al., 2005). A more recently introduced technique, digital tomosynthesis, generally delivers better results, mainly due to its three-dimensional (3D) imaging ability (multiple 2D slices through the breast) which reduces the effect of overlying breast tissue camouflaging focal breast masses (Ciatto et al., 2013). However, due to its inherent 
limitations, tomosynthesis cannot produce 3D images with the same image quality as computed tomography (CT) (Malliori et al., 2012). Therefore, it is important to investigate the opportunities for 3D mammographic CT imaging that could satisfy the requirements of medical practice in terms of the dose delivered to the patient, the image quality and the costs.

It has been shown recently (Zhao et al., 2012) that analyserbased CT (AB-CT) may allow 3D imaging of soft tissues and tumours at higher resolution and better contrast, and with a smaller radiation dose, compared with current clinical mammography. At the same time, recent theoretical and experimental studies (Diemoz et al., 2012; Gureyev et al., 2013, 2014a; Nesterets \& Gureyev, 2014) have shown that, depending on the specific parameters of the experiment, alternative $\mathrm{X}$-ray phase-contrast imaging methods, such as the propagation-based phase-contrast tomography (PB-CT), can deliver outcomes comparable with AB-CT with regard to image quality and dose, while being potentially simpler and cheaper to implement.

As a pre-requisite for successful translation of 3D phasecontrast mammography into clinical practice, it is essential to evaluate, quantify and optimize the main parameters of the PB-CT imaging technique, including the choice of X-ray energy, sample-to-detector distance, strategies for CT scans and the reconstruction techniques capable of maximizing the contrast-to-noise ratio (CNR) and suitable figures-of-merit (FOM) as a function of the X-ray dose delivered to the patient. While substantial progress has been achieved in this area in the last few years (Bravin et al., 2013; Olivo et al., 2013), there is still a great need for further research, testing and development before these techniques become suitable for routine clinical applications. The potential importance and value of optimized strategies for $\mathrm{CT}$ data acquisition and processing in phase-contrast imaging modalities have been clearly demonstrated in recent publications (Gureyev et al., 2013, 2014a; Nesterets \& Gureyev, 2014), unequivocally proving the existence of a significant potential for improvement in this field.

In our previous experiments at the SYRMEP beamline of the Elettra synchrotron in Trieste in 2013-2014 we performed multiple scans in AB-CT and PB-CT modes using plastic phantoms and several breast-tissue samples with normal and malignant tissues at different X-ray energies between 20 and $40 \mathrm{keV}$. These experiments have been very informative (Gureyev et al., 2013, 2014a; Pacilè et al., 2015), although an obvious limitation of these results was the inability to run similar tests at higher X-ray energies due to limitation on the energy range of the SYRMEP beamline. Note that according to some publications (e.g. Zhao et al., 2012) the use of highenergy X-rays (60 keV and higher) can lead to further reduction of the dose and improvement of the corresponding CNR and FOM in the case of mammographic AB-CT. However, the corresponding energy dependence in the case of PB-CT has not been fully demonstrated experimentally yet, as far as we know. This question has been addressed during our recent experiment (which was carried out at X-ray energies up to $50 \mathrm{keV}$ ) at the Imaging and Medical beamline of the
Australian Synchrotron. The results of this experiment are presented below.

\section{Experiment description}

We have conducted in-line phase-contrast CT imaging experiments at the Imaging and Medical beamline of the Australian Synchrotron (Stevenson et al., 2010). The detector used was a Hamamatsu CMOS Flat Panel Sensor C9252DK14 , utilized in partial scan mode, with pixel size $100 \mu \mathrm{m} \times$ $100 \mu \mathrm{m}, 1174 \times 99$ pixels $(\mathrm{H} \times \mathrm{V})$ field of view and 12-bit output. The detector has a CsI scintillator directly deposited on a $2 \mathrm{D}$ photodiode array.

A specially designed and fabricated phantom was used in this experiment. A CT slice of the phantom is shown in Fig. 1. The phantom consists of a cylindrical block made of polycarbonate, with a diameter of $10 \mathrm{~cm}$ and height of $2 \mathrm{~cm}$, having eight irregularly located cylindrical holes of $1 \mathrm{~cm}$ diameter, each filled with different substances as explained in the caption of Fig. 1. The chemical composition and mass density for the substances constituting the phantom are contained in Table 1.

The sample was imaged with monochromatic X-rays at three energies: $38 \mathrm{keV}, 45 \mathrm{keV}$ and $50 \mathrm{keV}$. While the sourceto-detector distance, $R$, was fixed to about $142.5 \mathrm{~m}$, the sample rotation-axis to detector distance (sample-to-detector distance, for short), $R_{2}$, was set to one of four values: $27 \mathrm{~cm}$, $1 \mathrm{~m}, 2 \mathrm{~m}$ and $5 \mathrm{~m}$. Table 2 lists the corresponding geometrical magnifications of the imaging setups, $M$, and the effective pixel size of the detector, $h$. Thus the total number of different imaging configurations was 12 . For each configuration, eight $360^{\circ}$ scans [four $180^{\circ}$ scans for two configurations, $(45 \mathrm{keV}$, $5 \mathrm{~m})$ and $(50 \mathrm{keV}, 5 \mathrm{~m})]$ were collected, with an angular step of approximately $0.1^{\circ}$. For each individual CT scan, 40 dark-field images and 40 flat-field images were collected, half before and half after a sample scan.

\section{Results and discussion}

CT data analysis (including pre-processing of data, CT reconstruction and optional post-processing) was carried out using X-TRACT software (X-TRACT, 2015; Gureyev et al., 2011; Thompson et al., 2011).

For each individual CT scan the pre-processing of image data consisted of three main steps: (i) zinger (hot pixel) filtering of all images, (ii) subtraction of the average dark field from the corresponding average flat field and from individual sample projections, and (iii) division of the dark-fieldcorrected individual sample projections by the dark-fieldcorrected average flat field. After pre-processing, the normalized projections were converted to sinograms.

$\mathrm{CT}$ reconstruction of the imaginary part, $\beta$, of the X-ray complex refractive index $n=1-\delta+i \beta$, unless otherwise stated, was carried out using a GPU-accelerated implementation (Nesterets \& Gureyev, 2009) of conventional parallel-beam filtered back-projection (FBP) algorithm utilizing a ramp filter. 
Table 1

Chemical composition and mass densities of the materials present or emulated in the phantom.

\begin{tabular}{|c|c|c|c|}
\hline \multirow{4}{*}{$\begin{array}{l}\text { Substance } \\
\text { Polycarbonate } \\
\text { Glycerol } \\
\text { Calcium chloride } 1 M\end{array}$} & \multicolumn{2}{|c|}{$\begin{array}{l}\text { Composition } \\
\text { (formula or weight \%) }\end{array}$} & \multirow{2}{*}{$\begin{array}{l}\text { Mass density } \\
\text { at } 293 \mathrm{~K}\left(\mathrm{~g} \mathrm{~cm}^{-3}\right) \\
1.2\end{array}$} \\
\hline & \multicolumn{2}{|c|}{$\mathrm{C}_{16} \mathrm{H}_{14} \mathrm{O}_{3}$} & \\
\hline & \multicolumn{2}{|c|}{$\mathrm{C}_{3} \mathrm{H}_{8} \mathrm{O}_{3}$} & \multirow{5}{*}{$\begin{array}{l}1.261 \\
1.086\end{array}$} \\
\hline & $\mathrm{Ca}$ & 3.69 & \\
\hline & $\mathrm{Cl}$ & 6.53 & \\
\hline & $\mathrm{H}$ & 10.05 & \\
\hline & $\mathrm{O}$ & 79.73 & \\
\hline \multirow[t]{3}{*}{ Ethanol 35v\% } & $\mathrm{H}$ & 11.75 & \multirow[t]{3}{*}{0.956} \\
\hline & $\mathrm{C}$ & 15.07 & \\
\hline & $\mathrm{O}$ & 73.18 & \\
\hline \multirow[t]{2}{*}{ Paraffin oil } & $\mathrm{H}$ & 14.86 & \multirow[t]{2}{*}{$0.827-0.890$} \\
\hline & $\mathrm{C}$ & 85.14 & \\
\hline Water & $\mathrm{H}_{2} \mathrm{O}$ & & 1 \\
\hline \multirow{8}{*}{ Gland tissue $\dagger$} & $\mathrm{H}$ & 10.2 & \multirow[t]{8}{*}{1.04} \\
\hline & $\mathrm{C}$ & 18.4 & \\
\hline & $\mathrm{N}$ & 3.2 & \\
\hline & $\mathrm{O}$ & 67.7 & \\
\hline & S & 0.125 & \\
\hline & $\mathrm{P}$ & 0.125 & \\
\hline & $\mathrm{K}$ & 0.125 & \\
\hline & $\mathrm{Ca}$ & 0.125 & \\
\hline \multirow[t]{8}{*}{ Adipose tissue $\dagger$} & $\mathrm{H}$ & 11.2 & \multirow[t]{8}{*}{0.93} \\
\hline & $\mathrm{C}$ & 61.9 & \\
\hline & $\mathrm{N}$ & 1.7 & \\
\hline & $\mathrm{O}$ & 25.1 & \\
\hline & S & 0.025 & \\
\hline & $\mathrm{P}$ & 0.025 & \\
\hline & $\mathrm{K}$ & 0.025 & \\
\hline & $\mathrm{Ca}$ & 0.025 & \\
\hline \multirow{8}{*}{$\begin{array}{l}\text { Glandular tissue } \\
\text { (50 w\% adipose, } \\
50 \mathrm{w} \% \text { glandular) }\end{array}$} & $\mathrm{H}$ & 10.7 & \multirow[t]{8}{*}{0.982} \\
\hline & $\mathrm{C}$ & 40.15 & \\
\hline & $\mathrm{N}$ & 2.45 & \\
\hline & $\mathrm{O}$ & 46.4 & \\
\hline & $\mathrm{P}$ & 0.075 & \\
\hline & S & 0.075 & \\
\hline & $\mathrm{K}$ & 0.075 & \\
\hline & $\mathrm{Ca}$ & 0.075 & \\
\hline
\end{tabular}

$\dagger$ Hammerstein et al. (1979).

The post-processing step consisted of ring-removal filtering of the reconstructed axial CT slices using an implementation of the algorithm proposed by Sijbers \& Postnov (2004).

A single representative axial CT slice was chosen (from the stack of 99 slices) for comparison of different experimental

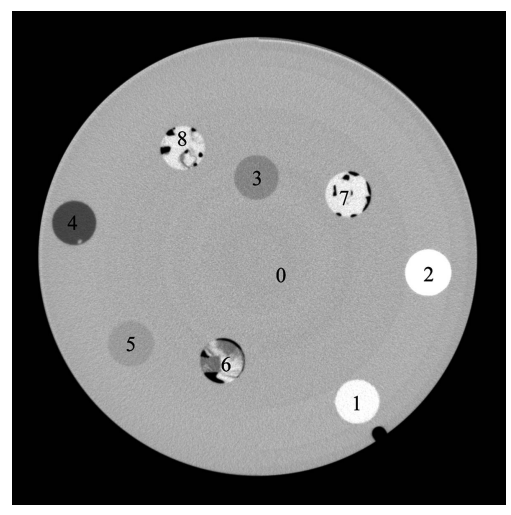

Figure 1

Composition of the phantom used in the experiment. 0: polycarbonate; 1: glycerol; 2: calcium chloride $(1 M)$; 3: ethanol $(35 \mathrm{v} \%)$; 4: paraffin oil; 5: water; 6: fatty ham; 7: meaty ham; 8 : fibrous ham.
Table 2

Geometrical parameters for different experimental configurations.

Values in parentheses represent errors in the least significant digit.

\begin{tabular}{llll}
\hline$R(\mathrm{~m})$ & $R_{2}(\mathrm{~m})$ & $M \dagger$ & $h \ddagger(\mu \mathrm{m})$ \\
\hline & 0.27 & $1.001898(7)$ & $99.8106(7)$ \\
$142.5(5)$ & 1 & $1.00707(3)$ & $99.298(2)$ \\
& 2 & $1.01423(5)$ & $98.597(5)$ \\
& 5 & $1.0364(1)$ & $96.49(1)$ \\
\hline
\end{tabular}

$\dagger M \equiv R /\left(R-R_{2}\right)$ is the geometrical magnification. $\$ h$ is the pixel size in the reconstructed slice. Due to magnification, this size is smaller than the detector pixel size.

Table 3

Values of the parameter $R_{\mathrm{abs}, r}$ used in dose calculations, for different parameters of the phantom shown in Fig. 4.

\begin{tabular}{lllll}
\hline & & \multicolumn{2}{l}{$R_{\text {abs }, r}$} \\
\cline { 3 - 5 }$R_{\text {out }}(\mathrm{cm})$ & $r(\mathrm{~cm})$ & $38 \mathrm{keV}$ & $45 \mathrm{keV}$ & $50 \mathrm{keV}$ \\
\hline 3 & 2.5 & 0.20271 & 0.14886 & 0.12579 \\
4 & 3.5 & 0.28496 & 0.21688 & 0.18557 \\
5 & 4.5 & 0.35841 & 0.27961 & 0.24279 \\
\hline
\end{tabular}

configurations and conditions. The results of the $\mathrm{CT}$ reconstruction process described above, for 12 experimental configurations, using all available projection data (see $\$ 2$ for details) are presented in Figs. 2 and 3. Detailed analysis of these results, in terms of the reconstruction accuracy, is carried out below, in $\S 3.2$. The effect of in-line phase contrast on CT reconstruction is discussed in $\$ 3.3$. In $\$ 3.4$, we evaluate the quality of CT reconstructions using several quality measures. In view of application to mammography, most of the quality measures are dose-normalized. For this reason, we begin our analysis by estimating, in $\$ 3.1$, the mean glandular dose (MGD) for individual projection images, for different experimental conditions.

\subsection{Radiation dose estimations}

Using the breast phantom schematically depicted in Fig. 4 (NCRP, 2004; Dance, 1990), the mean absorbed dose (in mGy) of the glandular tissue, having radius $r$ (in $\mathrm{cm}$ ) and located inside a cylinder of radius $R_{\text {out }}$ (in $\mathrm{cm}$ ), consisting of adipose tissue (fat), simulating the skin layer, can be calculated as follows (Johns \& Yaffe, 1985; Nesterets \& Gureyev, 2014):

$$
\begin{aligned}
& D_{\mathrm{abs}}[\mathrm{mGy}]=(2 / \pi) 1.602 \times 10^{-10}
\end{aligned}
$$

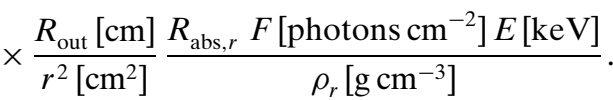

Here, $E$ is the $\mathrm{X}$-ray energy (in $\mathrm{keV}$ ), $F$ is the incident photon fluence (in photons $\mathrm{cm}^{-2}$ ), $\rho_{r}$ is the mass density of the glandular tissue (in $\mathrm{g} \mathrm{cm}^{-3}$ ) and $R_{\mathrm{abs}, r}$ is the fraction of X-ray energy incident on the phantom and absorbed in the glandular tissue. The latter was calculated using Monte Carlo (MC) simulations and the results are shown in Fig. 5 and in Table 3. The chemical compositions for the skin layer and glandular tissue used in the MC simulations are presented in Table 1. 


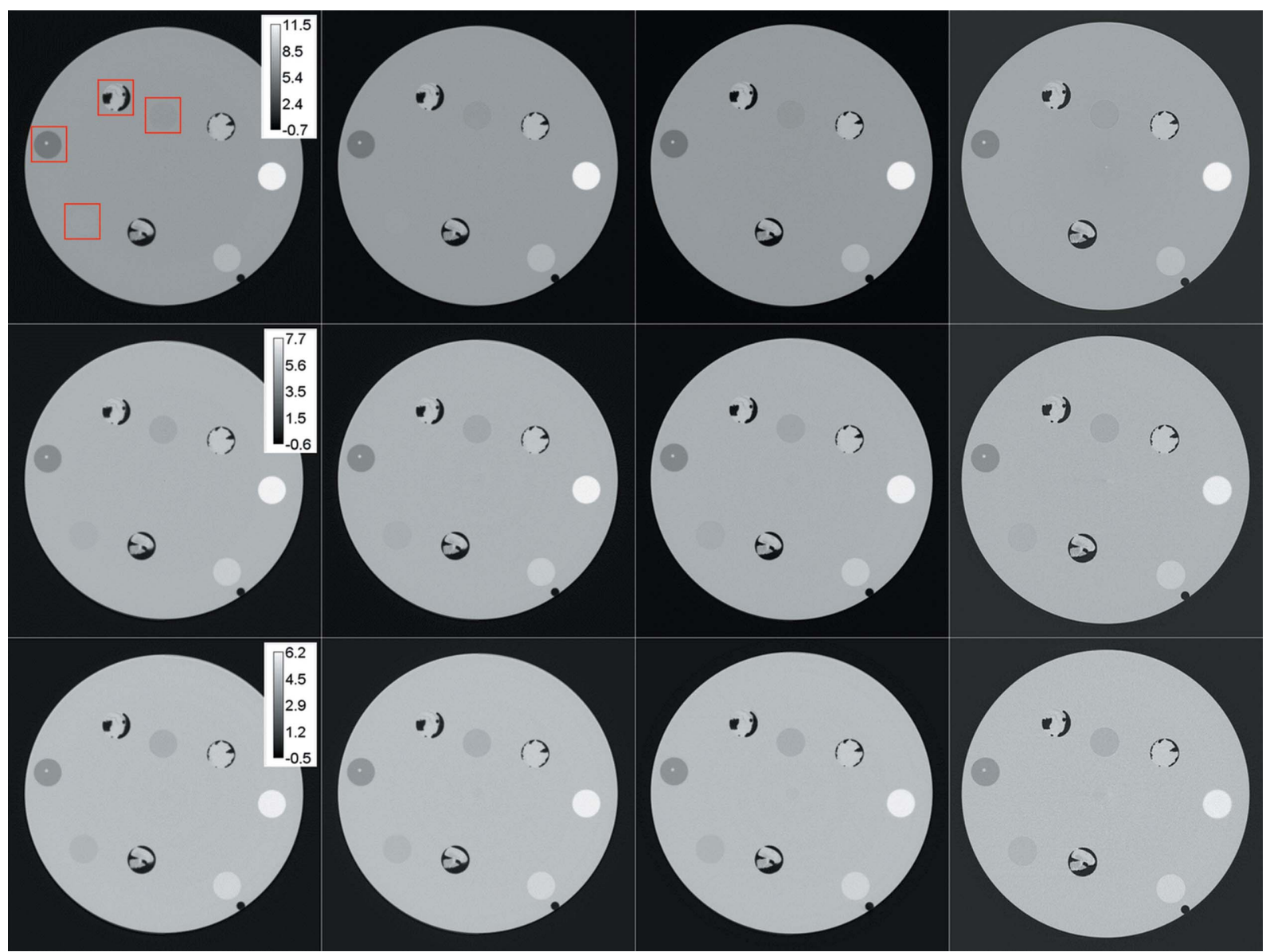

Figure 2

FBP CT reconstructions of the imaginary part, $\beta$, of the X-ray complex refractive index using the full sets of 28920 projections [7232 projections for two configurations, $(45 \mathrm{keV}, 5 \mathrm{~m})$ and $(50 \mathrm{keV}, 5 \mathrm{~m})$ ], for 12 combinations of the sample-to-detector distance and X-ray energy. Left to right: $0.27 \mathrm{~m}, 1 \mathrm{~m}, 2 \mathrm{~m}$, $5 \mathrm{~m}$; top to bottom: $38 \mathrm{keV}, 45 \mathrm{keV}, 50 \mathrm{keV}$. The calibration bars in the left-most plots show $\beta \times 10^{11}$.

During the experiment, an ion chamber (IC) was installed in the X-ray beam upstream from the sample, at a distance of about $136.2 \mathrm{~m}$ from the source. The readings from the IC were recorded during CT data acquisition. These readings have been used for measuring the photon fluence rate and the corresponding rate of the surface absorbed dose to air, $D R_{\mathrm{IC}}$, at the IC plane. Taking into account the exposure time of $33 \mathrm{~ms}$ for each individual projection, the photon fluences per individual projection image, in the IC plane, $F_{\mathrm{IC}}$, for each of 12 $\mathrm{CT}$ scans, have been calculated and the values are contained in the third column of Table 4. The latter values were used for calculating the corresponding incident photon fluences in the sample plane, $F_{\text {smpl }}$ (the third last column in Table 4 ), by taking into account the attenuation of the X-ray beam by an air gap between the IC and the sample, as well as the geometrical magnification of the X-ray beam between the IC and the sample. Similarly, the surface absorbed doses to air at the sample plane, $D_{\text {smpl, }}$, were calculated. Using equation (1), the photon fluences in the sample plane were converted into MGDs per individual projection image, for the phantom having inner and outer radii of $4.5 \mathrm{~cm}$ and $5 \mathrm{~cm}$, respectively. The calculated MGDs are contained in the last column of Table 4.
Comparison of the last two columns in Table 4 shows that the surface dose to air, $D_{\text {smpl }}$, is consistently bigger than the calculated MGD, especially for lower energies. This is partially due to the shielding effect of the skin layer and also due to the nature of the MGD which quantifies an average X-ray energy absorbed in the glandular tissue.

\subsection{Accuracy of the quantitative $\mathrm{CT}$ reconstruction}

Tables 5 and 6 show the theoretical, $\beta_{\text {theor }}$, and the experimental, $\beta_{\exp }$, values of the imaginary part of the complex refractive index, for materials constituting the phantom, for several selected experimental configurations. The experimental values were measured in CT slices reconstructed from the full set of 28920 projections (see \$2).

Analysis of the data contained in Tables 5 and 6 indicates that, in general, the experimentally measured $\beta$-values [and the associated linear attenuation coefficients $\mu \equiv(4 \pi / \lambda) \beta]$ are consistently smaller (by about 1-3\%) than the theoretical values. The only exception is the ethanol solution for which the experimental $\beta$-value is slightly larger than the theoretical one. This can be explained by a slightly smaller concentration of ethanol compared with the nominal concentration of 
Table 4

Incident photon fluences (per single projection) and the corresponding mean glandular absorbed doses calculated, using equation (1), for the cylindrical numerical phantom shown in Fig. 4 (with outer radius $R_{\text {out }}=5 \mathrm{~cm}$ and inner radius $r=4.5 \mathrm{~cm}$ ).

\begin{tabular}{|c|c|c|c|c|c|c|c|c|c|}
\hline $\begin{array}{l}\text { Energy } \\
(\mathrm{keV})\end{array}$ & $\begin{array}{l}R_{2} \\
(\mathrm{~m})\end{array}$ & $\begin{array}{l}F_{\mathrm{IC}}^{\dagger} \times 10^{-7} \\
(\text { photons cm } \\
\end{array}$ & $\begin{array}{l}D R_{\mathrm{IC}}^{\ddagger} \\
\left(\mathrm{mGy} \mathrm{s}^{-1}\right)\end{array}$ & $\begin{array}{l}\Delta z \S \\
(\mathrm{m})\end{array}$ & $T_{\text {air }}$ ฯ & $m+\dagger$ & $\begin{array}{l}F_{\text {smpp }}+\div \times 10^{-7} \\
\left(\text { photons cm } \mathrm{cm}^{-2} \text { ) }\right.\end{array}$ & $\begin{array}{l}D_{\text {smpl }} \S \S \\
(\mu \mathrm{Gy})\end{array}$ & $\begin{array}{l}D_{\text {abs }} \boldsymbol{\top} \uparrow \\
(\mu \mathrm{Gy})\end{array}$ \\
\hline \multirow[t]{3}{*}{38} & 0.27 & $8.59(3)$ & $1.250(4)$ & $6.0(5)$ & $0.83(1)$ & $1.044(4)$ & $6.5(1)$ & $31.3(5)$ & $22.8(4)$ \\
\hline & 1 & $8.29(3)$ & $1.208(4)$ & $5.3(5)$ & $0.85(1)$ & $1.039(4)$ & $6.5(1)$ & $31.2(5)$ & $22.7(4)$ \\
\hline & 5 & $9.10(3)$ & $1.390(5)$ & $1.3(5)$ & $0.96(2)$ & $1.010(4)$ & $8.6(2)$ & $43.2(8)$ & $30.0(5)$ \\
\hline \multirow[t]{2}{*}{45} & 0.27 & $6.12(2)$ & 0.775 (3) & $6.0(5)$ & $0.85(1)$ & $1.044(4)$ & $4.77(8)$ & $19.9(3)$ & $15.4(2)$ \\
\hline & 1 & $6.19(2)$ & 0.704 (3) & $5.3(5)$ & 0.87 (1) & 1.039 (4) & $4.96(8)$ & $18.6(3)$ & $16.0(3)$ \\
\hline \multirow{3}{*}{50} & 1 & $5.27(2)$ & $0.523(2)$ & $5.3(5)$ & 0.87 (1) & 1.039 (4) & 4.27 (6) & $14.0(2)$ & $13.3(2)$ \\
\hline & 2 & $5.33(2)$ & $0.531(2)$ & $4.3(5)$ & $0.90(1)$ & $1.032(4)$ & $4.49(7)$ & $14.8(2)$ & $14.0(2)$ \\
\hline & 5 & $6.14(2)$ & $0.610(2)$ & $1.3(5)$ & $0.97(1)$ & $1.010(4)$ & $5.83(9)$ & $19.1(3)$ & $18.2(3)$ \\
\hline
\end{tabular}

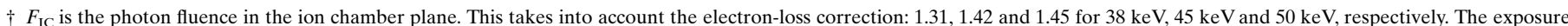

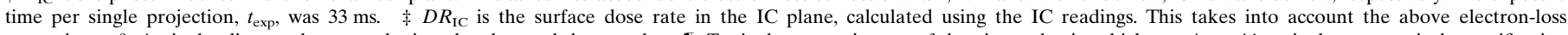

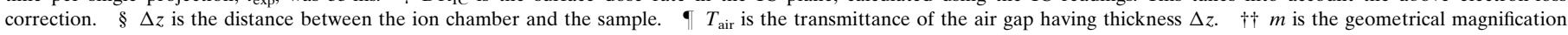
between the ion chamber plane (located at the distance $R_{\mathrm{IC}}=136.2 \mathrm{~m}$ from the source) and the sample plane (located at the distance $R_{\mathrm{IC}}+\Delta z$ from the source), $m=1+$

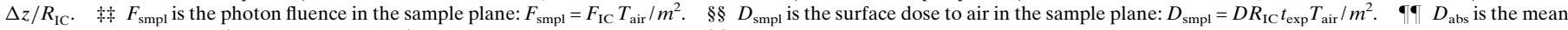
glandular absorbed dose (per single projection) and is calculated using equation (1) and the values of the parameter $R_{\mathrm{abs}, r}$ from the last row of Table 3 .

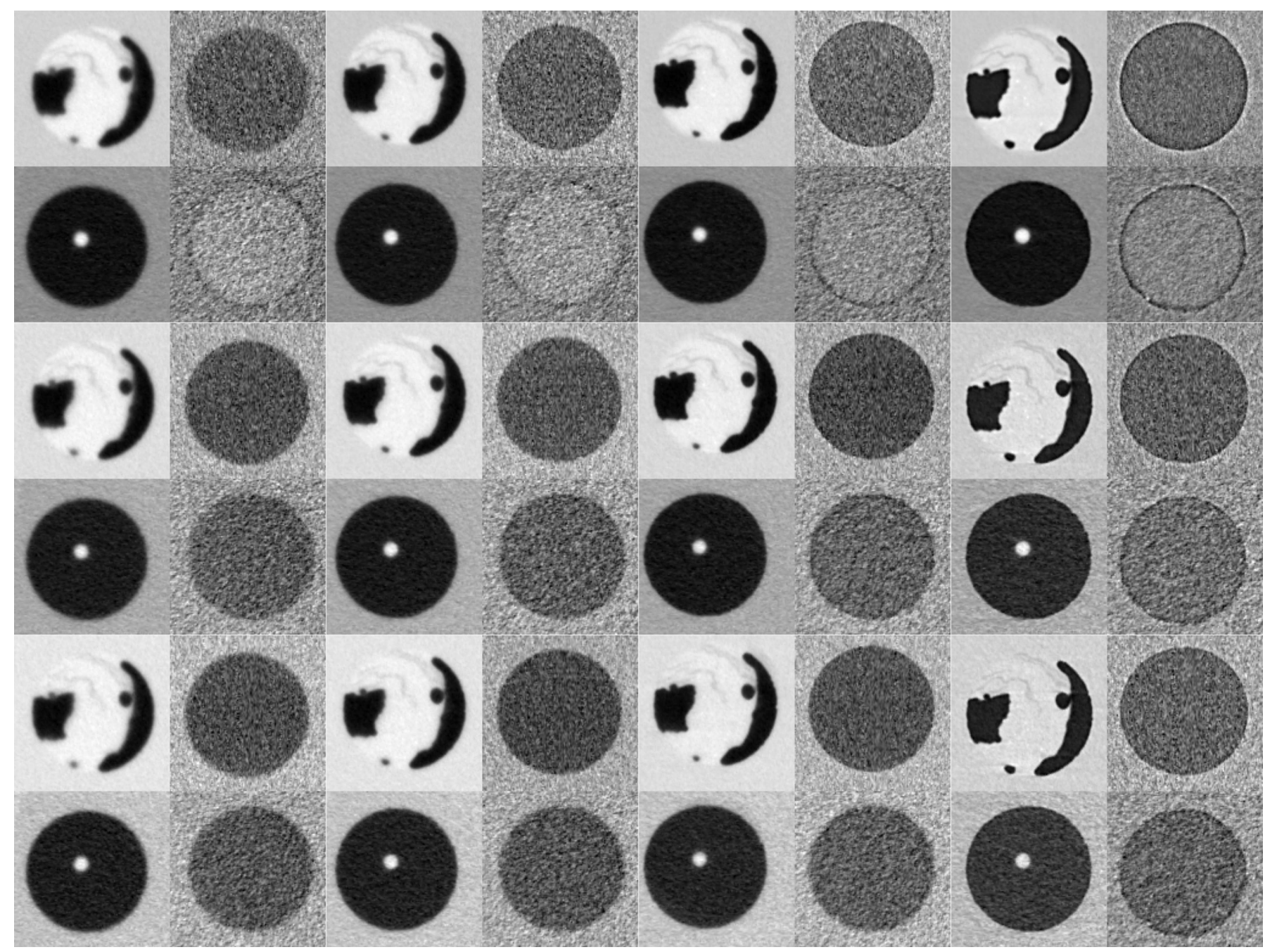

Figure 3

Enlarged fragments of the reconstructed slices shown in Fig. 2 corresponding to the inserts 8, 3, 4 and 5 of the phantom (see Fig. 1 for details) and indicated by square boxes shown in the top-left image in Fig. 2. For each fragment the image histogram was adjusted to the fragment's minimum and maximum values. 


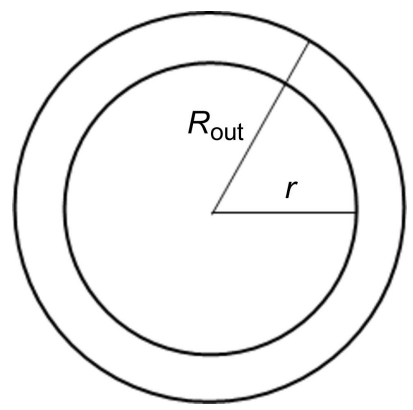

Figure 4

Schematic diagram of the numerical phantom used for mean glandular dose calculations.

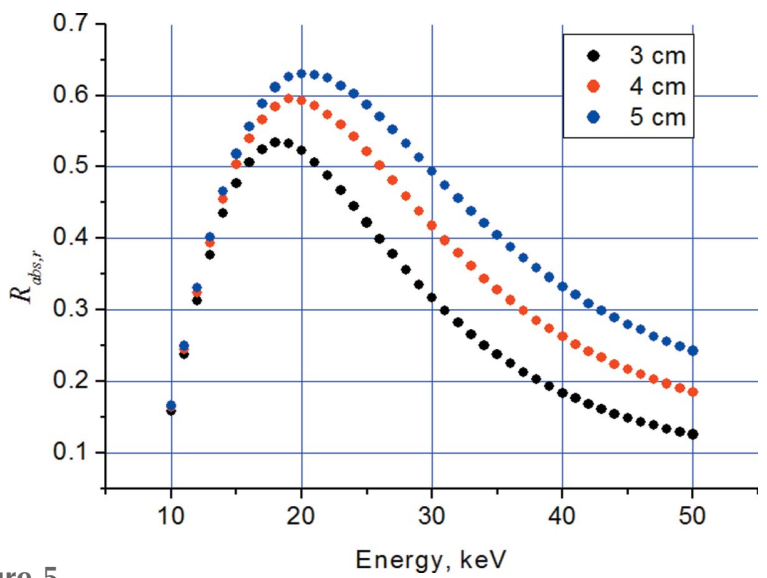

Figure 5

Energy dependencies of the parameter $R_{\mathrm{abs}, r}$ specifying the fraction of the X-ray energy incident on the phantom, shown in Fig. 4, and absorbed in the glandular tissue forming a cylinder of radius $r$, for different radii of the phantom (see also text in $\$ 3.1$ ).

35 volume percent $(35 \mathrm{v} \%)$. Also, for paraffin oil, the mass density, as provided by the manufacturer, was in the range $0.827-0.890 \mathrm{~g} \mathrm{~cm}^{-3}$. As a result, Tables 5 and 6 contain the corresponding ranges for the theoretical $\beta$-values, and the experimentally measured $\beta$-values are well within these ranges.

It is also worth mentioning that the $\beta$-values of polycarbonate were observed to slightly vary (by up to about $2 \%$ from the average value) across the reconstructed CT slices (note that in Tables 5 and 6 the values without brackets were measured near the centre of the reconstructed slices while the values in the square brackets were measured near the edge of the slices). Moreover, the degree of this non-uniformity was different for different experimental conditions (X-ray energy and/or sample-to-detector distance).

Fig. 6 shows experimental $\beta$-values obtained from CT scans collected at a sample-to-detector distance of $2 \mathrm{~m}$, plotted against the theoretical energy dependencies of the corresponding $\beta$-values, for some materials constituting the phantom.

\subsection{Phase contrast observation}

It should be emphasized that the $\mathrm{CT}$ slices presented in Fig. 2 were deliberately reconstructed without phase retrieval.

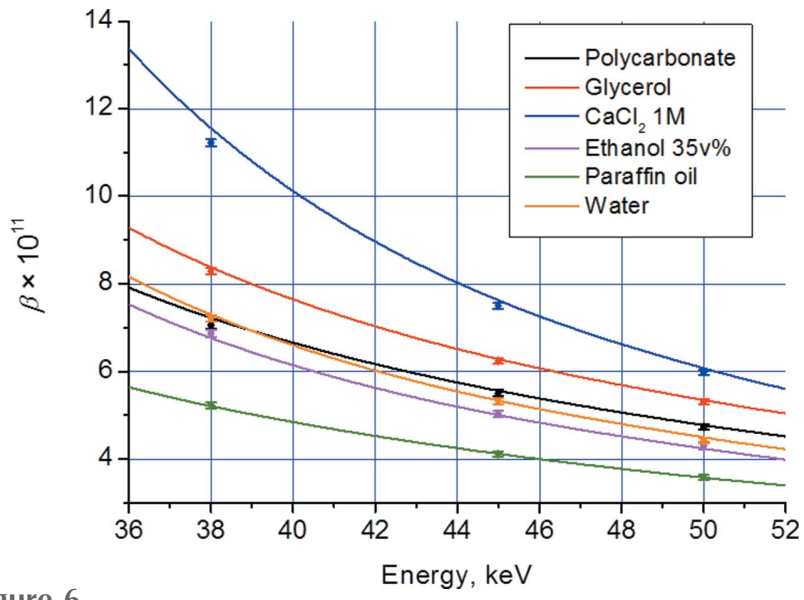

Figure 6

Theoretical (solid lines) and experimentally measured (dots) values of the imaginary part of the complex refractive index, for some materials constituting the phantom. Experimental points correspond to the full set of 28920 projections collected at a sample-to-detector distance of $2 \mathrm{~m}$. A mass density of $0.86 \mathrm{~g} \mathrm{~cm}^{-3}$ was used for paraffin oil (see Table 1).

As a result, in-line phase contrast manifests itself as edge enhancement in these slices. In order to make it easier for the reader to observe this effect, Fig. 3 shows enlarged fragments of the slices delineated in the top-left image of Fig. 2 using square boxes. Analysis of Fig. 3 indicates that in the case of the smallest sample-to-detector distance $(0.27 \mathrm{~m})$ the features of the phantom have visibly smeared edges (see the first column in Fig. 3). By increasing the sample-to-detector distance, one can first clearly observe edge sharpness improvement (see the second and third columns in Fig. 3) and eventually the appearance of phase-contrast fringes which are most pronounced at $5 \mathrm{~m}$ sample-to-detector distance (see the fourth column in Fig. 3).

Whereas the phase contrast increases with increasing sample-to-detector distance, it decreases with increasing X-ray energy. This can be explained using the transport of intensity equation (TIE) (Teague, 1983):

$$
\begin{aligned}
I_{R^{\prime}}(x, y ; \lambda)= & I_{0}(x, y ; \lambda) \\
& -R^{\prime} \lambda /(2 \pi) \nabla \cdot\left[I_{0}(x, y ; \lambda) \nabla \varphi_{0}(x, y ; \lambda)\right],
\end{aligned}
$$

where $I_{R^{\prime}}(x, y ; \lambda)$ and $I_{0}(x, y ; \lambda)=I_{\text {in }}(x, y ; \lambda) \exp [-(4 \pi / \lambda)$ $\left.\int \beta(x, y, z ; \lambda) \mathrm{d} z\right]$ are the intensity distributions in the image and the object planes, respectively, $\varphi_{0}(x, y ; \lambda)=\varphi_{\text {in }}(x, y ; \lambda)-$ $(2 \pi / \lambda) \int \delta(x, y, z ; \lambda) \mathrm{d} z$ is the phase distribution in the object plane, $I_{\text {in }}(x, y ; \lambda)$ and $\varphi_{\text {in }}(x, y ; \lambda)$ are the intensity and phase distributions in the illuminating beam, and $R^{\prime}$ is the effective propagation distance. According to the TIE, the magnitude of the phase contrast at any boundary is proportional to the effective propagation distance $R^{\prime}$ (which, in the case of large source-to-sample distances, is accurately approximated by the sample-to-detector distance $R_{2}$ ) and to the real decrement $\left(\delta_{1}-\delta_{2}\right)$ of the relative complex refractive index for two materials forming the boundary. For hard X-rays, the real decrement $\delta$ of the refractive index for any material decreases with increasing X-ray energy. Analysis of Figs. 2 and 3 shows that, as expected, the phase-contrast effects become weaker 
Table 5

Theoretical, $\beta_{\text {theor }}$, and experimental, $\beta_{\text {exp }}$, values of the imaginary part of the complex refractive index for materials constituting the phantom (see Fig. 1 for details), for the three $\mathrm{X}$-ray energies used in the experiment.

The experimental values were measured in CT slices reconstructed from the full set of 28920 projections collected at a sample-to-detector distance of $2 \mathrm{~m}$. For polycarbonate, values without brackets were measured near the centre of the reconstructed slices while values in square brackets were measured near the edge of the slices.

\begin{tabular}{|c|c|c|c|c|c|c|c|c|c|c|}
\hline \multirow[b]{2}{*}{ Index } & \multirow[b]{2}{*}{ Material } & \multicolumn{3}{|l|}{$E=38 \mathrm{keV}$} & \multicolumn{3}{|l|}{$E=45 \mathrm{keV}$} & \multicolumn{3}{|l|}{$E=50 \mathrm{keV}$} \\
\hline & & $\beta_{\text {theor }} \times 10^{11}$ & $\beta_{\exp } \times 10^{11}$ & $\beta_{\exp } / \beta_{\text {theor }}$ & $\beta_{\text {theor }} \times 10^{11}$ & $\beta_{\exp } \times 10^{11}$ & $\beta_{\exp } / \beta_{\text {theor }}$ & $\beta_{\text {theor }} \times 10^{11}$ & $\beta_{\exp } \times 10^{11}$ & $\beta_{\text {exp }} / \beta_{\text {theor }}$ \\
\hline 0 & Polycarbonate & 7.225 & $\begin{array}{l}7.1(1) \\
{[7.18(8)]}\end{array}$ & $\begin{array}{l}0.99(2) \\
{[0.99(1)]}\end{array}$ & 5.560 & $\begin{array}{l}5.49(8) \\
{[5.52(6)]}\end{array}$ & $\begin{array}{l}0.99(2) \\
{[0.99(1)]}\end{array}$ & 4.774 & $\begin{array}{l}4.72(7) \\
{[4.74(6)]}\end{array}$ & $\begin{array}{l}0.99(2) \\
{[0.99(1)]}\end{array}$ \\
\hline 1 & Glycerol & 8.380 & $8.29(8)$ & 0.99 (1) & 6.283 & $6.24(7)$ & 0.99 (1) & 5.346 & $5.31(6)$ & 0.99 (1) \\
\hline 2 & $\mathrm{CaCl}_{2} 1 M$ & 11.557 & $11.18(9)$ & $0.968(8)$ & 7.625 & $7.50(7)$ & $0.983(9)$ & 6.073 & $5.98(6)$ & $0.99(1)$ \\
\hline 3 & Ethanol 35v\% & 6.764 & 6.9 (1) & $1.02(2)$ & 5.004 & $5.10(8)$ & $1.02(2)$ & 4.237 & $4.30(7)$ & $1.01(2)$ \\
\hline 4 & Paraffin oil & $5.007-5.389$ & $5.23(7)$ & - & $3.960-4.261$ & $4.11(6)$ & - & $3.436-3.698$ & $3.57(6)$ & - \\
\hline 7 & Meaty ham & - & $8.5(1)$ & - & - & $6.12(9)$ & - & - & $5.06(7)$ & - \\
\hline 8 & Fibrous ham & - & $8.5(1)$ & - & - & $6.04(9)$ & - & - & $5.01(7)$ & - \\
\hline
\end{tabular}

Table 6

Theoretical, $\beta_{\text {theor }}$, and experimental, $\beta_{\text {exp }}$, values of the imaginary part of the complex refractive index for materials constituting the phantom (see Fig. 1 for details), for three sample-to-detector distances.

The experimental values were measured in CT slices reconstructed from the full set of 28920 projections collected at an X-ray energy of $38 \mathrm{keV}$. For polycarbonate, values without brackets were measured near the centre of the reconstructed slices while values in square brackets were measured near the edge of the slices.

\begin{tabular}{|c|c|c|c|c|c|c|c|c|}
\hline \multirow[b]{2}{*}{ Index } & \multirow[b]{2}{*}{ Material } & \multirow[b]{2}{*}{$\beta_{\text {theor }} \times 10^{11}$} & \multicolumn{2}{|l|}{$R_{2}=0.27 \mathrm{~m}$} & \multicolumn{2}{|l|}{$R_{2}=1 \mathrm{~m}$} & \multicolumn{2}{|l|}{$R_{2}=5 \mathrm{~m}$} \\
\hline & & & $\beta_{\exp } \times 10^{11}$ & $\beta_{\exp } / \beta_{\text {theor }}$ & $\beta_{\exp } \times 10^{11}$ & $\beta_{\exp } / \beta_{\text {theor }}$ & $\beta_{\exp } \times 10^{11}$ & $\beta_{\exp } / \beta_{\text {theor }}$ \\
\hline 0 & Polycarbonate & 7.225 & 7.0 (1) $[7.14(7)]$ & $0.97(1)[0.99(1)]$ & $7.2(1)[7.17(8)]$ & $0.99(1)[0.99(1)]$ & 7.0 (1) $[7.18(8)]$ & $0.97(2)[0.99(1)]$ \\
\hline 2 & $\mathrm{CaCl}_{2} 1 M$ & 11.557 & $11.09(9)$ & $0.960(8)$ & $11.21(8)$ & $0.970(7)$ & $11.20(9)$ & $0.969(8)$ \\
\hline 3 & Ethanol 35v\% & 6.764 & $6.81(9)$ & $1.01(1)$ & $6.9(1)$ & $1.02(1)$ & $6.8(1)$ & $1.01(2)$ \\
\hline 4 & Paraffin oil & $5.007-5.389$ & $5.21(7)$ & - & $5.23(7)$ & - & $5.22(7)$ & - \\
\hline 5 & Water & 7.296 & $7.17(8)$ & $0.98(1)$ & $7.23(8)$ & $0.99(1)$ & $7.20(9)$ & $0.99(1)$ \\
\hline 8 & Fibrous ham & - & $8.3(1)$ & - & $8.4(1)$ & - & $8.5(1)$ & - \\
\hline
\end{tabular}

when the X-ray energy increases. In particular, this becomes apparent if one compares fragments in the top element of the right-most column with the corresponding fragments of the other two elements of that column (the rows correspond to X-ray energy of $38 \mathrm{keV}$, $45 \mathrm{keV}$ and $50 \mathrm{keV}$, respectively).

Remarkably, in-line phase contrast, clearly observed at a sample-to-detector distance of $5 \mathrm{~m}$, is quite significant despite the relatively large effective pixel size of the detector, about $100 \mu \mathrm{m}$, and the large horizontal source size, about $800 \mu \mathrm{m}$ (note, however, that the effective source size in the detector plane is significantly smaller, by a factor of about 5/142). In order to quantify the effect of in-line phase contrast on the sharpness of the edges of the phantom features we measured their width using the following approach. First we selected linear profiles across the boundaries between the inserts and the polycarbonate cylinder in CT slices which were four-fold oversampled using linear interpolations. Then, these profiles were fitted with a sigmoidal function resulting from a convolution of

Table 7
Widths (in $\mu \mathrm{m}$ ) of the boundaries between polycarbonate and three selected materials, in CT slices reconstructed using the FBP algorithm, applied to the full set of 28920 projections at $38 \mathrm{keV}$, and with post-reconstruction ring filtering. phase-retrieved projections (TIE-HOM with $\gamma=1000$ ).

\begin{tabular}{lllll}
\hline & Width $(\mu \mathrm{m})$ & & \\
\cline { 2 - 5 } Material & $R_{2}=0.27 \mathrm{~m}$ & $R_{2}=1 \mathrm{~m}$ & $R_{2}=2 \mathrm{~m}$ & $R_{2}=5 \mathrm{~m}$ \\
\hline Glycerol & $526 \pm 20$ & $323 \pm 19(371 \pm 15)$ & $333 \pm 20(399 \pm 12)$ & $242 \pm 16(414 \pm 9)$ \\
CaCl $_{2} 1 M$ & $499 \pm 8$ & $468 \pm 7(495 \pm 4)$ & $477 \pm 6(553 \pm 4)$ & $492 \pm 7(626 \pm 3)$ \\
Paraffin oil & $425 \pm 9$ & $347 \pm 8(382 \pm 6)$ & $344 \pm 9(425 \pm 6)$ & $185 \pm 8(355 \pm 4)$ \\
\hline
\end{tabular}

a sharp edge profile with a Gaussian point spread function (PSF), $f(x)=a+b \operatorname{erf}\left[(x-c) /\left(2^{1 / 2} \sigma\right)\right](a, b, c$ and $\sigma$ are the fitting parameters). The full width at half-maximum (FWHM) of the Gaussian PSF, $2.3548 \sigma$, was considered as the width of the edge.

The widths of the boundaries between polycarbonate and three selected materials, i.e. glycerol, calcium chloride and paraffin oil, are given in Table 7. As expected, the width of the edges in the reconstructed CT slices usually decreases with increasing sample-to-detector distance (Gureyev et al., 2004). 
Moreover, for a fixed sample-to-detector distance, the edge sharpness improvement strongly depends on the X-ray optical properties of the neighbouring materials, namely, on the ratio $\left(\delta_{1}-\delta_{2}\right) /\left(\beta_{1}-\beta_{2}\right)$. Theoretical values for this ratio, for several material combinations, are provided in Table 8. In particular, amongst the three materials, the edge sharpness improvement was the biggest for paraffin oil, for which the ratio was the biggest. On the other hand, for calcium chloride, for which the ratio is negative, the edge sharpness was almost constant with increasing sample-to-detector distance.

\subsection{Effect of phase retrieval on the quality of CT reconstruction}

It is well acknowledged that phase retrieval using an algorithm based on the homogeneous transport of intensity equation (TIE-HOM) (Paganin et al., 2002),

$$
I_{R^{\prime}}(x, y ; \lambda)=\left[1-R^{\prime} \lambda \gamma /(4 \pi) \nabla^{2}\right] I_{0}(x, y ; \lambda),
$$

results, in general, in reduced noise in reconstructed CT slices while preserving the sharpness of the edges when used with a proper parameter $\gamma$ quantifying the relationship between the real and imaginary parts of the decrement of the refraction index of the object (see, for example, Beltran et al., 2011; Nesterets \& Gureyev, 2014). We applied the TIE-HOM algorithm [which corresponds to inversion of equation (3)] to projection data sets and subsequently carried out FBP CT reconstruction. In order to restrict the absorbed dose to values currently accepted for standard mammographic screening, we restricted the number of projections to 361 over $180^{\circ}$ (this is an 80 -fold reduction compared with the high photon statistics data used for CT reconstructions shown in Figs. 2 and 3). The
Table 8

Values of the ratio $\left(\delta_{1}-\delta_{2}\right) /\left(\beta_{1}-\beta_{2}\right)$ for different material combinations and $\mathrm{X}$-ray energies of interest.

\begin{tabular}{llll}
\hline & \multicolumn{2}{l}{ Ratio $\left(\delta_{1}-\delta_{2}\right) /\left(\beta_{1}-\beta_{2}\right)$} \\
\cline { 2 - 4 } Material combination & $E=38 \mathrm{keV}$ & $E=45 \mathrm{keV}$ & $E=50 \mathrm{keV}$ \\
\hline Glycerol/polycarb. & 1302 & 1483 & 1519 \\
$\mathrm{CaCl}_{2} /$ polycarb. & -245 & -368 & -474 \\
Ethanol 35v\%/polycarb. & 6176 & 3649 & 3066 \\
Paraffin oil $\dagger$ /polycarb. & 1992 & 1987 & 1933 \\
Water/polycarb. & -31098 & 6951 & 4592 \\
Polycarb./air & 2516 & 2331 & 2199 \\
Gland/adipose & 1083 & 1268 & 1328 \\
\hline
\end{tabular}

$\dagger$ Mass density $0.86 \mathrm{~g} \mathrm{~cm}^{-3}$ was used in calculations.

MGDs, $D$, per complete CT scans, were calculated using the dose values per single projection, from the last column of Table 4, and multiplying them by the number of projections in a CT scan. For a 361-projection CT scan, $D$ was between $4.7 \mathrm{mGy}$ and $10.8 \mathrm{mGy}$, depending on the imaging parameters.

In this paper, our primary target is mammographic CT. In the case of breast tissue its main components are gland and adipose tissues. According to Table 8 , in the $\mathrm{X}$-ray energy range from $38 \mathrm{keV}$ to $50 \mathrm{keV}$ the ratio $\left(\delta_{1}-\delta_{2}\right) /\left(\beta_{1}-\beta_{2}\right)$ for the gland/adipose pair is slightly larger than 1000 . For this reason, the parameter $\gamma$ in the TIE-HOM-based phaseretrieval algorithm was set to 1000 for all three energies. One reconstructed CT slice, corresponding to an X-ray energy of $38 \mathrm{keV}$, for four values of the sample-to-detector distance, is shown in Fig. 7 together with its magnified fragments.

In order to quantify the quality of the reconstructions in the presence of noise we utilized the quality index recently introduced by Gureyev et al. $(2014 a, b)$. The results of this

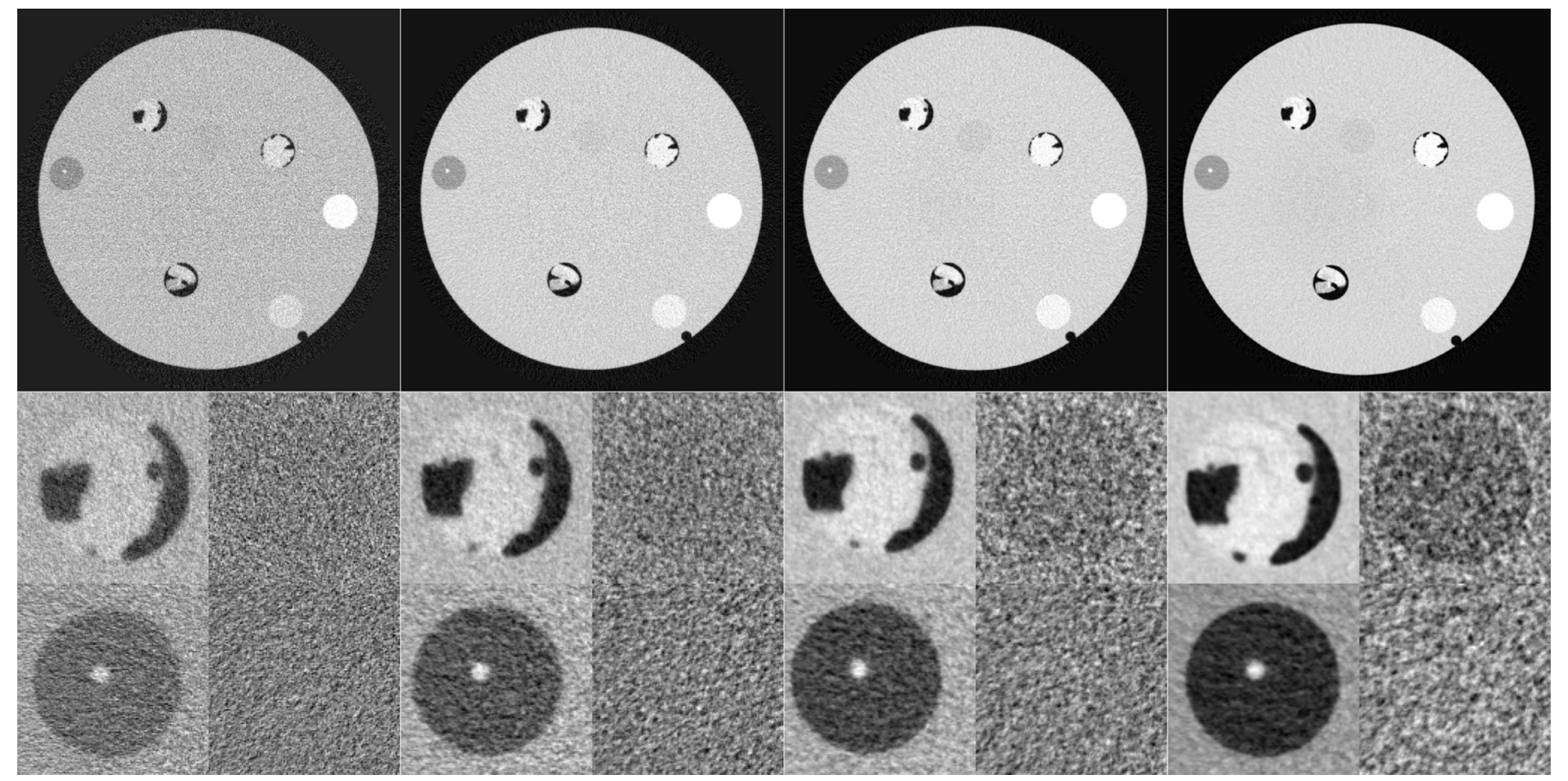

Figure 7

Effect of phase retrieval (TIE-HOM with $\gamma=1000)$ on FBP CT reconstruction from low-photon-statistics data $\left(361\right.$ projections over $\left.180^{\circ}\right)$, at $38 \mathrm{keV}$ X-ray energy, for different sample-to-detector distances. From left to right: $0.27 \mathrm{~m}, 1 \mathrm{~m}, 2 \mathrm{~m}$ and $5 \mathrm{~m}$. 
Table 9

Quality characteristics of CT slices reconstructed (using FBP) from phase-retrieved (using TIE-HOM with $\gamma=1000$ ) low-photon-statistics (361 projections) data at X-ray energy $E=38 \mathrm{keV}$, for different sample-to-detector distances.

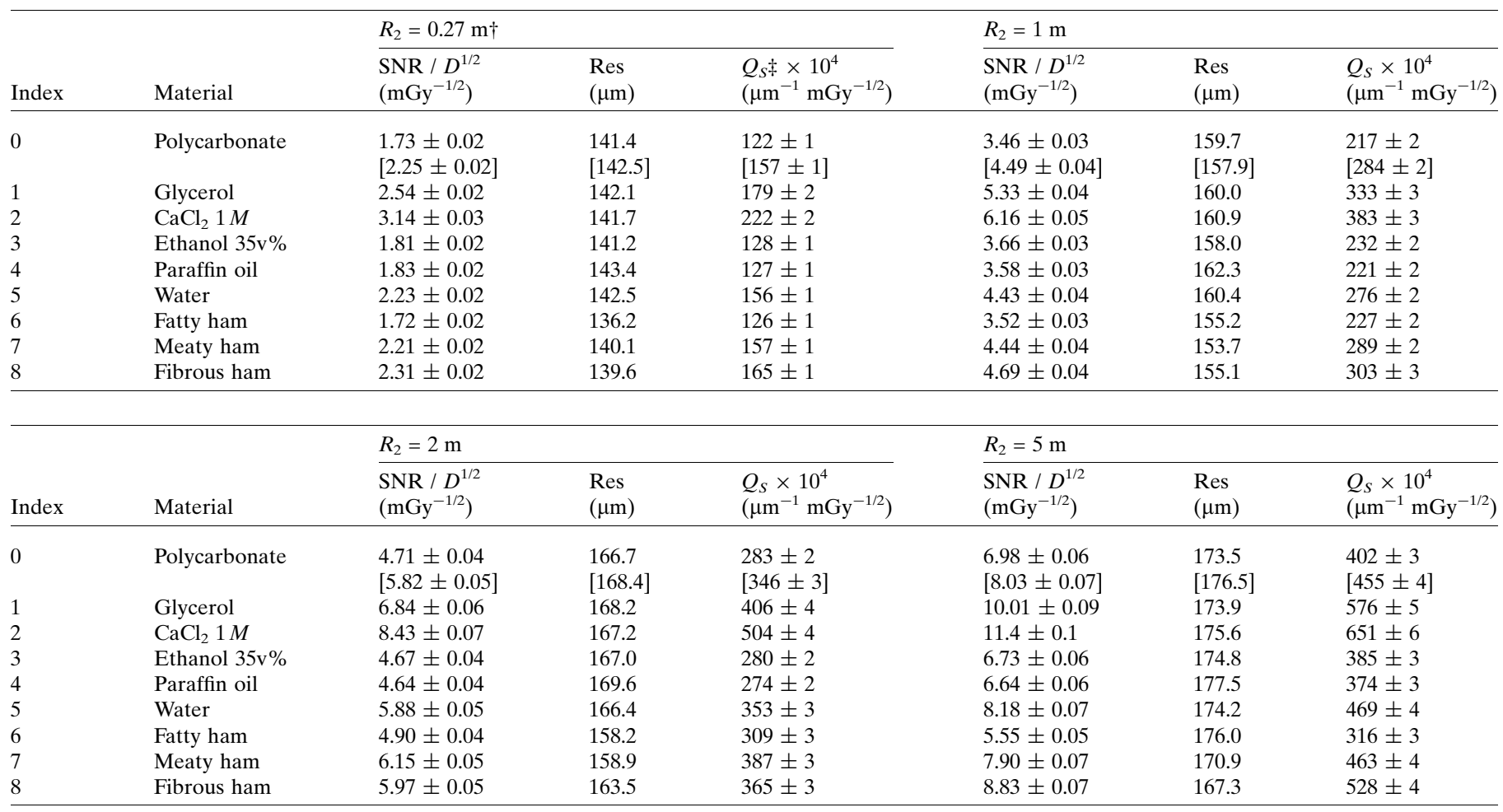

$\dagger$ No phase retrieval. $\neq Q_{S}=\mathrm{SNR} / D^{1 / 2} /$ Res.

analysis are summarized in Tables 9 and 10 which contain values of three parameters characterizing the image quality including the signal-to-noise ratio (SNR) per unit dose, SNR/ $D^{1 / 2}$, the spatial resolution and the ratio of the two which we use as the modified quality index, $Q_{S}$, in this paper.

Both the SNR and the spatial resolution have been calculated using a uniform region inside each feature of the phantom. The SNR was defined as the ratio of the mean $\beta$ value in the region to its standard deviation. The resolution was estimated by calculating the noise power spectrum in the region and evaluating the inverse of its second moment's square root. Note that the thus defined resolution can only be used to accurately estimate the characteristic length scale of spatially correlated noise. This characteristic of noise is complementary to its standard deviation and, in general, these two cannot be improved simultaneously (see, for example, Gureyev et al., 2014b). We should emphasize that it is this definition of spatial resolution that is used below, in our quantitative analysis.

We also calculated the conventional contrast-to-noise ratio (CNR),

$$
\mathrm{CNR}=\frac{\left|\left\langle\beta_{1}\right\rangle-\left\langle\beta_{2}\right\rangle\right|}{\left\{\left[\operatorname{var}\left(\beta_{1}\right)+\operatorname{var}\left(\beta_{2}\right)\right] / 2\right\}^{1 / 2}},
$$

which quantifies the ability to differentiate materials in the sample. Here the angular brackets and $\operatorname{var}(\ldots)$ designate the mean value and the variance of a spatial distribution, respectively. In our subsequent data analysis we evaluate the CNR per unit dose, $\mathrm{CNR} / D^{1 / 2}$, and (where available) the width of the boundaries between polycarbonate and inserts, FWHM, as well as the figure-of-merit, FOM, defined as the ratio of the CNR per unit dose to the width of the boundaries.

First, we investigate the effect of the sample-to-detector distance on the quality of $\mathrm{CT}$ slices reconstructed with the conventional FBP algorithm, using the quality measures defined above.

Analysis of the data in Table 9 shows that, with increasing sample-to-detector distance (within the range used in the experiment), the SNR per unit dose as well as the quality index are monotonically increasing, for all materials constituting the phantom. For SNR this is an expected behaviour due to the nature of the TIE-HOM algorithm. Indeed, the latter acts as a low-pass filter and by increasing the sample-todetector distance one achieves stronger suppression of high spatial frequencies in reconstructed projection images and hence stronger reduction of the standard deviation of noise in individual projections as well as in CT reconstructed slices (Nesterets \& Gureyev, 2014). It should be emphasized that this reduction of the standard deviation of noise comes with increase of the spatial correlation length of noise in reconstructed images, i.e. the system resolution degrades (Gureyev et al., 2013, 2014b). This is clearly observed in Table 9 by analysing the columns containing the values of the spatial resolution (Res). Note, however, that the relative degradation 
Table 10

Quality characteristics of CT slices reconstructed (using FBP) from phase-retrieved (using TIE-HOM with $\gamma=1000$ ) low-photon-statistics (361 projections) data at the sample-to-detector distance $R_{2}=5 \mathrm{~m}$, for different X-ray energies.

The values in bold indicate the best result for each quality characteristic.

\begin{tabular}{|c|c|c|c|c|c|c|c|c|c|c|}
\hline \multirow[b]{2}{*}{ Index } & \multirow[b]{2}{*}{ Material } & \multicolumn{3}{|l|}{$E=38 \mathrm{keV}$} & \multicolumn{3}{|l|}{$E=45 \mathrm{keV}$} & \multicolumn{3}{|l|}{$E=50 \mathrm{keV}$} \\
\hline & & $\begin{array}{l}\mathrm{SNR} / D^{1 / 2} \\
\left(\mathrm{mGy}^{-1 / 2}\right)\end{array}$ & $\begin{array}{l}\text { Res } \\
(\mu \mathrm{m})\end{array}$ & $\begin{array}{l}Q_{S}^{\dagger} \times 10^{4} \\
\left(\mu \mathrm{m}^{-1} \mathrm{mGy}^{-1 / 2}\right)\end{array}$ & $\begin{array}{l}\mathrm{SNR} / D^{1 / 2} \\
\left(\mathrm{mGy}^{-1 / 2}\right)\end{array}$ & $\begin{array}{l}\text { Res } \\
(\mu \mathrm{m})\end{array}$ & $\begin{array}{l}Q_{S} \times 10^{4} \\
\left(\mu \mathrm{m}^{-1} \mathrm{mGy}^{-1 / 2}\right)\end{array}$ & $\begin{array}{l}\mathrm{SNR} / D^{1 / 2} \\
\left(\mathrm{mGy}^{-1 / 2}\right)\end{array}$ & $\begin{array}{l}\text { Res } \\
(\mu \mathrm{m})\end{array}$ & $\begin{array}{l}Q_{S} \times 10^{4} \\
\left(\mu \mathrm{m}^{-1} \mathrm{mGy}^{-1 / 2}\right)\end{array}$ \\
\hline 0 & Polycarbonate & $\begin{array}{l}6.98 \pm 0.06 \\
{[8.03 \pm 0.07]}\end{array}$ & $\begin{array}{l}173.5 \\
{[176.5]}\end{array}$ & $\begin{array}{l}402 \pm 3 \\
{[455 \pm 4]}\end{array}$ & $\begin{array}{l}\mathbf{7 . 3 1} \pm 0.06 \\
{[\mathbf{8 . 5 7} \pm 0.06]}\end{array}$ & $\begin{array}{l}173.2 \\
{[175.3]}\end{array}$ & $\begin{array}{l}\mathbf{4 2 2} \pm 3 \\
{[\mathbf{4 8 9} \pm 4]}\end{array}$ & $\begin{array}{l}7.09 \pm 0.05 \\
{[8.10 \pm 0.06]}\end{array}$ & $\begin{array}{l}171.6 \\
{[174.0]}\end{array}$ & $\begin{array}{l}413 \pm 3 \\
{[465 \pm 3]}\end{array}$ \\
\hline 1 & Glycerol & $\mathbf{1 0 . 0 1} \pm 0.09$ & 173.9 & $576 \pm 5$ & $9.59 \pm 0.07$ & 175.8 & $546 \pm 4$ & $8.64 \pm 0.06$ & 175.1 & $494 \pm 4$ \\
\hline 2 & $\mathrm{CaCl}_{2} 1 M$ & $\mathbf{1 1 . 4} \pm 0.1$ & 175.6 & $\mathbf{6 5 1} \pm 6$ & $10.74 \pm 0.08$ & 174.6 & $616 \pm 5$ & $9.40 \pm 0.07$ & 176.6 & $533 \pm 4$ \\
\hline 3 & Ethanol 35v\% & $6.73 \pm 0.06$ & 174.8 & $385 \pm 3$ & $6.76 \pm 0.05$ & 173.7 & $389 \pm 3$ & $6.39 \pm 0.05$ & 173.4 & $369 \pm 3$ \\
\hline 4 & Paraffin oil & $6.64 \pm 0.06$ & 177.5 & $374 \pm 3$ & $\mathbf{7 . 3 0} \pm 0.06$ & 174.2 & $421 \pm 3$ & $6.86 \pm 0.05$ & 174.5 & $394 \pm 3$ \\
\hline 5 & Water & $\mathbf{8 . 1 8} \pm 0.07$ & 174.2 & $469 \pm 4$ & $8.09 \pm 0.06$ & 175.5 & $461 \pm 3$ & $7.66 \pm 0.05$ & 173.6 & $442 \pm 3$ \\
\hline 6 & Fatty ham & $5.55 \pm 0.05$ & 176.0 & $316 \pm 3$ & $\mathbf{7 . 3 1} \pm 0.06$ & 166.1 & $440 \pm 3$ & $6.84 \pm 0.05$ & 170.5 & $401 \pm 3$ \\
\hline 7 & Meaty ham & $7.90 \pm 0.07$ & 170.9 & $463 \pm 4$ & $\mathbf{9 . 1 3} \pm 0.07$ & 166.1 & $\mathbf{5 4 9} \pm 4$ & $8.14 \pm 0.06$ & 168.4 & $484 \pm 3$ \\
\hline 8 & Fibrous ham & $8.83 \pm 0.07$ & 167.3 & $528 \pm 4$ & $\mathbf{9 . 1 0} \pm 0.07$ & 169.8 & $\mathbf{5 3 6} \pm 4$ & $8.46 \pm 0.06$ & 168.8 & $501 \pm 4$ \\
\hline
\end{tabular}

$\dagger Q_{S}=\mathrm{SNR} / D^{1 / 2} /$ Res.

of the resolution (with respect to the values corresponding to the shortest sample-to-detector distance of $0.27 \mathrm{~m}$ ) is small (about $25 \%$, for the longest sample-to-detector distance of $5 \mathrm{~m}$ ); phase retrieval is only one of several factors affecting the spatial resolution in a CT reconstructed volume. This explains, to some extent, the observed increase of the quality index with increasing sample-to-detector distance.

Although not presented in this paper, a behaviour similar to that of the SNR per unit dose was also observed for the CNR per unit dose. Namely, with increasing sample-to-detector distance the CNR per unit dose increased, due to the abovementioned effect of the TIE-HOM phase-retrieval algorithm. This is because the area contrast in CT reconstructed slices is independent of the sample-to-detector distance, while the standard deviation of noise (after TIE-HOM retrieval) decreases with increasing sample-to-detector distance and, according to equation (4), the CNR increases. Also, we observed an improvement of the FOM (the ratio of the CNR per unit dose to the FWHM of the material boundaries) for the selected materials (glycerol, calcium chloride and paraffin oil) with increasing sample-to-detector distance. This is explained by the observed behaviour of the FWHM of the boundaries between the selected materials and polycarbonate (see Table 7 for details). In particular, Table 7 indicates that in the case of glycerol and paraffin oil the width of the boundaries in CT slices reconstructed using phase-retrieved projections (the values in the parentheses) is usually smaller than in the CT slices reconstructed from contact projections (i.e. the projections collected at the sample-to-detector distance of $0.27 \mathrm{~m}$ ). In the case of calcium chloride, although the width of its boundaries with polycarbonate slightly increases with increasing sample-to-detector distance, by at most $25 \%$ with respect to the boundary width in CT slices reconstructed from contact projections, the FOM is still significantly increasing with increasing sample-to-detector distance (for the distances used in this experiment). Since the longest sample-to-detector distance of $5 \mathrm{~m}$ resulted in the best quality of $\mathrm{CT}$ reconstructed slices (in terms of the quality measures utilized in the paper), in our subsequent analysis we restrict our consideration to the case of a sample-to-detector distance of $5 \mathrm{~m}$.

We also investigated the dependence of the CT reconstruction quality on the X-ray energy, by comparing values of the above-defined quality measures for CT slices reconstructed using data collected at three X-ray energies: $38 \mathrm{keV}$, $45 \mathrm{keV}$ and $50 \mathrm{keV}$.

Analysis of data in Table 10 shows that, for the three X-ray energies used in the experiment, the SNR per unit dose as well as the quality index are monotonically decreasing for glycerol, calcium chloride and water with increasing X-ray energy. For other materials, both quality measures are maximal at the $\mathrm{X}$-ray energy of $45 \mathrm{keV}$. It is worth mentioning that the observed excellent correlation between the SNR per unit dose and the quality index [which is the ratio of the SNR per unit dose to the system resolution defined above, before equation (4)] can be explained by the fact that the measured system resolution is observed to be independent of the X-ray energy.

Regarding the quality of CT reconstructed slices in terms of the FOM, this can be improved (with respect to CT slices reconstructed from contact projections) using two alternative approaches: (i) by using raw (i.e. without phase retrieval) projections collected at a finite sample-to-detector distance, or (ii) by applying TIE-HOM phase retrieval to projection data. In the former case, the CNR is essentially independent of the sample-to-detector distance and the gain in the FOM (with respect to the CT slices reconstructed from contact projections) is totally due to improvement of the boundary sharpness as a result of in-line phase contrast (see Table 11). In the latter case, the CNR is significantly improved as a result of low-pass filtering of projections by the TIE-HOM algorithm (see also discussion above) while the sharpness of the boundaries, compared with the case of CT slices reconstructed from contact projections, is essentially preserved or degraded insignificantly (see Table 12). Comparison of data contained in Tables 11 and 12 allowed us to conclude that, in terms of the FOM and for the chosen value of the parameter $\gamma$ in the TIEHOM algorithm, the second approach is advantageous and 
Table 11

Quality characteristics of CT slices reconstructed (using FBP) from raw (without phase retrieval) low-photon-statistics (361 projections) data at the sample-to-detector distance $R_{2}=5 \mathrm{~m}$, for different X-ray energies.

The values in bold indicate the best result for each quality characteristic.

\begin{tabular}{|c|c|c|c|c|c|c|c|c|c|}
\hline \multirow[b]{2}{*}{$\begin{array}{l}\text { Material } \\
\text { combination }\end{array}$} & \multicolumn{3}{|l|}{$E=38 \mathrm{keV}$} & \multicolumn{3}{|l|}{$E=45 \mathrm{keV}$} & \multicolumn{3}{|l|}{$E=50 \mathrm{keV}$} \\
\hline & $\begin{array}{l}\mathrm{CNR} / D^{1 / 2} \\
\left(\mathrm{mGy}^{-1 / 2}\right)\end{array}$ & $\begin{array}{l}\text { FWHM } \\
(\mu \mathrm{m})\end{array}$ & $\begin{array}{l}\mathrm{FOM} \dagger \times 10^{4} \\
\left(\mu \mathrm{m}^{-1}\right. \\
\left.\mathrm{mGy}^{-1 / 2}\right)\end{array}$ & $\begin{array}{l}\mathrm{CNR} / D^{1 / 2} \\
\left(\mathrm{mG}^{-1 / 2}\right)\end{array}$ & $\begin{array}{l}\text { FWHM } \\
(\mu \mathrm{m})\end{array}$ & $\begin{array}{l}\mathrm{FOM} \times 10^{4} \\
\left(\mu \mathrm{m}^{-1}\right. \\
\left.\mathrm{mGy}^{-1 / 2}\right)\end{array}$ & $\begin{array}{l}\mathrm{CNR} / D^{1 / 2} \\
\left(\mathrm{mG}^{-1 / 2}\right)\end{array}$ & $\begin{array}{l}\text { FWHM } \\
(\mu \mathrm{m})\end{array}$ & $\begin{array}{l}\mathrm{FOM} \times 10^{4} \\
\left(\mu \mathrm{m}^{-1}\right. \\
\left.\mathrm{mGy}^{-1 / 2}\right)\end{array}$ \\
\hline Glycerol/polycarb. & $0.311 \pm 0.003$ & $242 \pm 16$ & $12.8 \pm 0.8$ & $\mathbf{0 . 3 1 2} \pm 0.002$ & $138 \pm 22$ & $\mathbf{2 3} \pm 4$ & $0.286 \pm 0.002$ & $207 \pm 34$ & $14 \pm 2$ \\
\hline $\mathrm{CaCl}_{2} /$ polycarb. & $\mathbf{1 . 0 5 9} \pm 0.009$ & $492 \pm 7$ & $21.6 \pm 0.4$ & $0.797 \pm 0.006$ & $385 \pm 17$ & $20.7 \pm 0.9$ & $0.593 \pm 0.004$ & $442 \pm 27$ & $13.5 \pm 0.8$ \\
\hline Ethanol/polycarb. & $0.0646 \pm 0.0005$ & - & - & $0.1531 \pm 0.0012$ & - & - & $0.1933 \pm 0.0014$ & - & - \\
\hline Paraffin/polycarb. & $0.583 \pm 0.005$ & $185 \pm 8$ & $31.5 \pm 1.4$ & $\mathbf{0 . 6 5 1} \pm 0.005$ & $138 \pm 13$ & $\mathbf{4 7} \pm 4$ & $0.624 \pm 0.004$ & $168 \pm 14$ & $37 \pm 3$ \\
\hline Water/polycarb. & $0.0057 \pm 0.0005$ & - & - & $0.0943 \pm 0.0007$ & - & - & $0.1173 \pm 0.0008$ & - & - \\
\hline Polycarb./air & $2.35 \pm 0.02$ & - & - & $2.62 \pm 0.02$ & - & - & $2.62 \pm 0.02$ & - & - \\
\hline Fatty/meaty ham & $0.589 \pm 0.005$ & - & - & $0.573 \pm 0.004$ & - & - & $0.522 \pm 0.004$ & - & - \\
\hline
\end{tabular}

$\dagger \mathrm{FOM}=\mathrm{CNR} / D^{1 / 2} / \mathrm{FWHM}$.

Table 12

Quality characteristics of CT slices reconstructed (using FBP) from phase-retrieved (using TIE-HOM with $\gamma=1000$ ) low-photon-statistics (361 projections) data at sample-to-detector distance $R_{2}=5 \mathrm{~m}$, for different X-ray energies.

The values in bold indicate the best result for each quality characteristic.

\begin{tabular}{|c|c|c|c|c|c|c|c|c|c|}
\hline \multirow[b]{2}{*}{$\begin{array}{l}\text { Material } \\
\text { combination }\end{array}$} & \multicolumn{3}{|l|}{$E=38 \mathrm{keV}$} & \multicolumn{3}{|l|}{$E=45 \mathrm{keV}$} & \multicolumn{3}{|l|}{$E=50 \mathrm{keV}$} \\
\hline & $\begin{array}{l}\mathrm{CNR} / D^{1 / 2} \\
\left(\mathrm{mGy}^{-1 / 2}\right)\end{array}$ & $\begin{array}{l}\text { FWHM } \\
(\mu \mathrm{m})\end{array}$ & $\begin{array}{l}\mathrm{FOM} \dagger \times 10^{4} \\
\left(\mu \mathrm{m}^{-1}\right. \\
\left.\mathrm{mGy}^{-1 / 2}\right)\end{array}$ & $\begin{array}{l}\mathrm{CNR} / D^{1 / 2} \\
\left(\mathrm{mGy}^{-1 / 2}\right)\end{array}$ & $\begin{array}{l}\text { FWHM } \\
(\mu \mathrm{m})\end{array}$ & $\begin{array}{l}\mathrm{FOM} \dagger \times 10^{4} \\
\left(\mu \mathrm{m}^{-1}\right. \\
\left.\mathrm{mGy}^{-1 / 2}\right)\end{array}$ & $\begin{array}{l}\mathrm{CNR} / D^{1 / 2} \\
\left(\mathrm{mGy}^{-1 / 2}\right)\end{array}$ & $\begin{array}{l}\text { FWHM } \\
(\mu \mathrm{m})\end{array}$ & $\begin{array}{l}\mathrm{FOM}^{1} \times 10^{4} \\
\left(\mu \mathrm{m}^{-1}\right. \\
\left.\mathrm{mGy}^{-1 / 2}\right)\end{array}$ \\
\hline Glycerol/polycarb. & $\mathbf{1 . 3 5 7} \pm 0.012$ & $414 \pm 9$ & $32.7 \pm 0.8$ & $1.120 \pm 0.008$ & $279 \pm 16$ & $\mathbf{4 0} \pm 2$ & $0.965 \pm 0.007$ & $481 \pm 25$ & $21 \pm 1$ \\
\hline $\mathrm{CaCl}_{2} /$ polycarb. & $\mathbf{4 . 2 4} \pm 0.04$ & $626 \pm 3$ & $67.7 \pm 0.7$ & $2.92 \pm 0.02$ & $559 \pm 10$ & $52 \pm 1$ & $2.011 \pm 0.014$ & $528 \pm 12$ & $38.1 \pm 0.9$ \\
\hline Ethanol/polycarb. & $0.262 \pm 0.002$ & - & - & $0.538 \pm 0.004$ & - & - & $0.664 \pm 0.005$ & - & - \\
\hline Paraffin/polycarb. & $\mathbf{2 . 4 5} \pm 0.02$ & $355 \pm 4$ & $69.1 \pm 0.9$ & $2.37 \pm 0.02$ & $338 \pm 7$ & $\mathbf{7 0} \pm 2$ & $2.10 \pm 0.02$ & $363 \pm 10$ & $58 \pm 2$ \\
\hline Water/polycarb. & $0.0170 \pm 0.0002$ & - & - & $0.318 \pm 0.002$ & - & - & $0.437 \pm 0.003$ & - & - \\
\hline Polycarb./air & $\mathbf{9 . 6 6} \pm 0.08$ & $260 \pm 2$ & $372 \pm 4$ & $9.45 \pm 0.07$ & $261 \pm 4$ & $363 \pm 6$ & $8.83 \pm 0.06$ & $251 \pm 4$ & $352 \pm 6$ \\
\hline Fatty ham/meaty ham & $2.42 \pm 0.02$ & - & - & $2.17 \pm 0.02$ & - & - & $1.69 \pm 0.01$ & - & - \\
\hline
\end{tabular}

$\dagger \mathrm{FOM}=\mathrm{CNR} / D^{1 / 2} / \mathrm{FWHM}$.

results in up to two- to three-fold improvement of the FOM compared with the first approach.

Also, for three selected materials, including glycerol, calcium chloride and paraffin oil (for which the CNR is relatively large), and for both approaches described above, the optimal X-ray energy that maximizes the FOM is $45 \mathrm{keV}$, in most cases. This behaviour correlates well with the general trend observed for the energy dependence of the quality index $Q_{S}$.

It is worth mentioning that analysis of Table 11 indicates that none of the material pairs (except for polycarbonate and air) can be reliably differentiated when using a threshold of 5 for the CNR (Rose, 1948) and a reasonable MGD of $4 \mathrm{mGy}$ (in order to obtain the CNR for this dose, one needs to multiply the values of $\mathrm{CNR} / D^{1 / 2}$ by two). On the other hand, analysis of Table 12 shows that TIE-HOM phase retrieval of projections prior to the FBP CT reconstruction results in significant, three- to four-fold, improvement of the CNR. In this case, two materials, calcium chloride and paraffin, can be reliably separated from polycarbonate, at least for the lowest of the three X-ray energies used.

Importantly, analysis of data in Tables 11 and 12 indicates that, in general, the energy dependence of the CNR per unit dose is not uniform. For the X-ray energies used in our experiment, one can easily reveal three typical behaviours: CNR is (i) decreasing with increasing X-ray energy, which is observed, for example, for glycerol and calcium chloride in polycarbonate and for the pair fatty ham/meaty ham, (ii) increasing with increasing X-ray energy, which is observed for ethanol and water in polycarbonate, and (iii) maximum at the $45 \mathrm{keV}$ energy, which is observed in CT slices reconstructed from raw projections for paraffin oil in polycarbonate. This observation indicates that the optimum X-ray energy that maximizes the CNR per unit dose and the FOM, in general, depends on the choice of the pair of materials. For mammographic application of CT, we expect that fatty ham and meaty ham better represent real breast tissue, compared with other materials in the phantom. As already discussed above, for this pair of tissues, and amongst the three X-ray energies used, the CNR is maximal at $38 \mathrm{keV}$ while the SNR as well as the quality index are maximal at $45 \mathrm{keV}$.

\subsection{Comparison of different $\mathrm{CT}$ reconstruction algorithms}

We investigated the possibility of improving the quality of CT reconstructions by utilizing four iterative CT reconstruction algorithms, including equal-slope tomography (EST) (Miao et al., 2005; X-TRACT, 2015), iterative FBP (Myers et 
Table 13

Quality characteristics of CT slices reconstructed (using alternative CT reconstruction algorithms) from phase-retrieved (using TIE-HOM with $\gamma=1000$ ) low-photon-statistics (361 projections, except for EST which used 340 projections) data at sample-to-detector distance $R_{2}=5 \mathrm{~m}$ and X-ray energy $E=38 \mathrm{keV}$.

EST (five iterations of gradient algorithm). iFBP (nBins $=512, \mathrm{NSR}=0.004$, HistPower-1 $=0$, Filter size $=3$ pixels, beta threshold $=0$ ). TV $($ regularization parameter $\beta=1, \mathrm{NSR}=0.004,10$ iterations). $\mathrm{RL}$ (NSR $=0.004,200$ iterations). The values in bold indicate the best result for each quality characteristic.

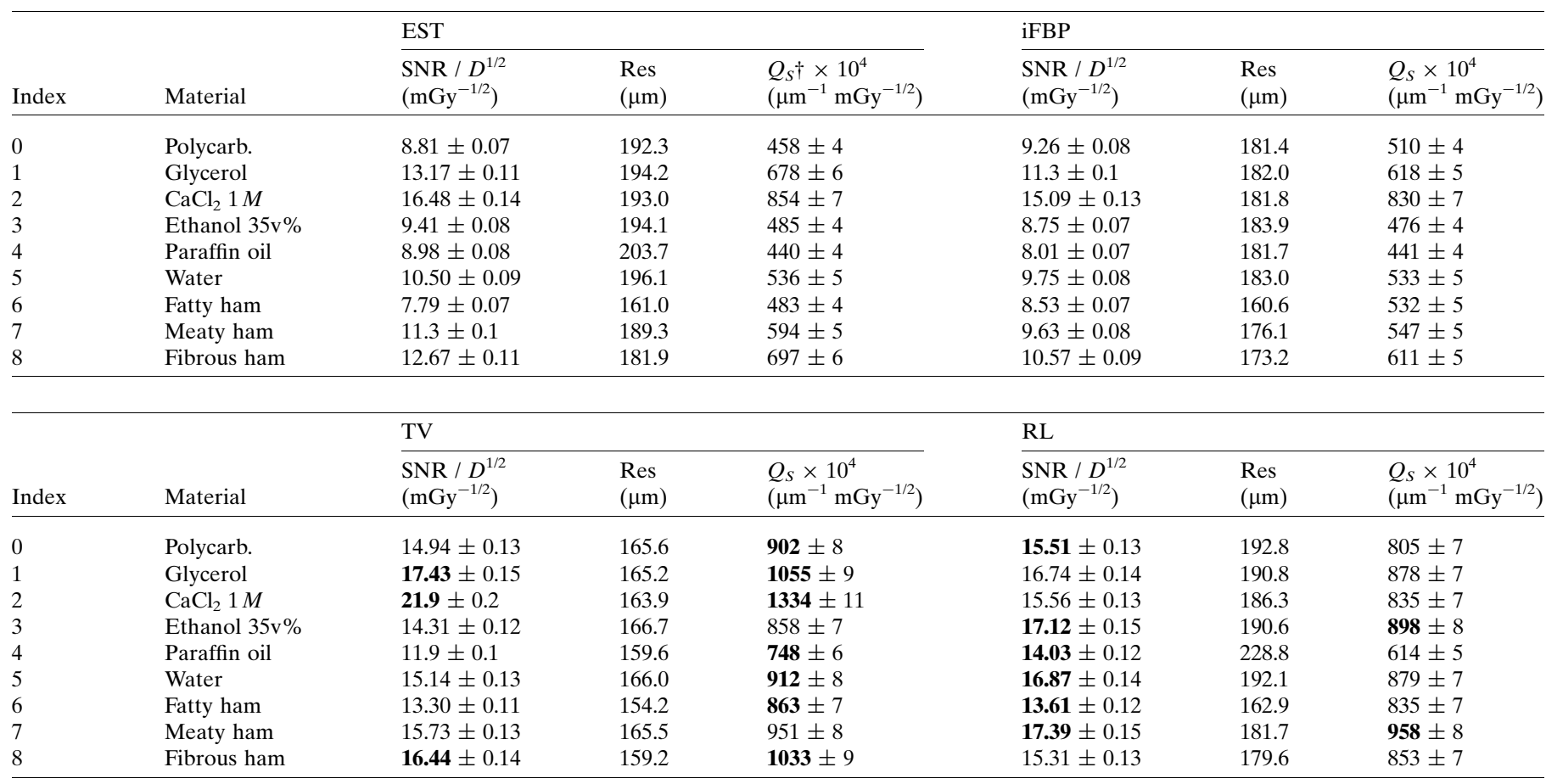

$\dagger Q_{S}=\mathrm{SNR} / D^{1 / 2} /$ Res

al., 2010; X-TRACT, 2015), an implementation of the total variation (TV) based algorithm (X-TRACT, 2015) and an implementation of the generic Richardson-Lucy (RL) reconstruction algorithm (Richardson, 1972; Lucy, 1974; X-TRACT, 2015). These algorithms have been applied to a single low-photon-statistics projection data set, consisting of 340 projections (with MGD of about $10.2 \mathrm{mGy}$ ) in the case of EST and 361 projections (with MGD of about $10.8 \mathrm{mGy}$ ) for other algorithms. The data set corresponds to an X-ray energy of $38 \mathrm{keV}$ and the longest sample-to-detector distance of $5 \mathrm{~m}$. TIE-HOM phase retrieval with the parameter $\gamma=1000$ has been applied to individual projections prior to $\mathrm{CT}$ reconstructions.

Reconstructed slices, together with their magnified fragments, are shown in Fig. 8. SNR per unit dose, the spatial resolution and the quality index $Q_{S}$ are provided in Table 13. CNR per unit dose, edge sharpness and FOM (where available) are given in Table 14. Below we provide brief comparisons, in terms of the quality measures used throughout the paper, of each of the reconstruction algorithms with the conventional FBP algorithm.

EST. Compared with the conventional FBP reconstruction algorithm, this method results in noticeable improvement of most quality measures, including the SNR, the quality index $Q_{S}$ and the FOM. It also seems to preserve the sharpness of the boundaries but noticeably degrades the spatial resolution. Visually, the reconstructed slices look slightly less noisy.
iFBP. Compared with the conventional FBP reconstruction algorithm, this method performs better in all respects: it improves the SNR and the quality index while only slightly degrading the spatial resolution; it also improves the CNR and the FOM and definitely outperforms the conventional FBP in terms of the boundary sharpness. Compared with EST, iFBP is generally better in terms of boundary sharpness, FOM and spatial resolution but is worse in terms of noise suppression and, as a result, is generally poorer in terms of the SNR and the quality index.

$T V$. Compared with the conventional FBP reconstruction algorithm, this method shows significant improvements in all respects. Moreover, this method is advantageous in terms of the quality index and the FOM; these are consistently maximal amongst the considered methods. At the same time, this method preserves and even improves the sharpness of boundaries. However, visual inspection of Fig. 8 indicates that this method results in a blocky structure of noise, despite the finest resolution as seen in Table 13 .

$R L$. This method outperforms the conventional FBP reconstruction algorithm in terms of noise suppression, but at the expense of spatial resolution and edge sharpness. It performs significantly better in terms of SNR, quality index and CNR but only slightly better in terms of FOM. Compared with other considered algorithms, this method is undoubtedly the best in terms of the SNR per unit dose and CNR per unit dose (see Tables 13 and 14). However, on account of system 
Table 14

Quality characteristics of CT slices reconstructed (using alternative CT reconstruction algorithms) from phase-retrieved (using TIE-HOM with $\gamma=1000$ ) low-photon-statistics (361 projections except for EST which used 340 projections) data at the sample-to-detector distance $R_{2}=5 \mathrm{~m}$ and X-ray energy $E=38 \mathrm{keV}$.

The values in bold indicate the best result for each quality characteristic.

\begin{tabular}{|c|c|c|c|c|c|c|}
\hline \multirow[b]{2}{*}{$\begin{array}{l}\text { Material } \\
\text { combination }\end{array}$} & \multicolumn{3}{|l|}{ EST } & \multicolumn{3}{|l|}{ iFBP } \\
\hline & $\begin{array}{l}\mathrm{CNR} / D^{1 / 2} \\
\left(\mathrm{mGy}^{-1 / 2}\right)\end{array}$ & $\begin{array}{l}\text { FWHM } \\
(\mu \mathrm{m})\end{array}$ & $\begin{array}{l}\mathrm{FOM} \dagger \times 10^{4} \\
\left(\mu \mathrm{m}^{-1} \mathrm{mGy}^{-1 / 2}\right)\end{array}$ & $\begin{array}{l}\mathrm{CNR} / D^{1 / 2} \\
\left(\mathrm{mGy}^{-1 / 2}\right)\end{array}$ & $\begin{array}{l}\text { FWHM } \\
(\mu \mathrm{m})\end{array}$ & $\begin{array}{l}\mathrm{FOM} \times 10^{4} \\
\left(\mu \mathrm{m}^{-1} \mathrm{mGy}^{-1 / 2}\right)\end{array}$ \\
\hline Glycerol/polycarb. & $1.77 \pm 0.02$ & $383 \pm 60$ & $46 \pm 7$ & $1.523 \pm 0.013$ & $238 \pm 85$ & $64 \pm 22$ \\
\hline $\mathrm{CaCl}_{2} /$ polycarb. & $5.96 \pm 0.05$ & $553 \pm 17$ & $108 \pm 3$ & $5.51 \pm 0.05$ & $557 \pm 20$ & $99 \pm 4$ \\
\hline Ethanol/polycarb. & $0.452 \pm 0.004$ & - & - & $0.401 \pm 0.003$ & - & - \\
\hline Paraffin/polycarb. & $3.27 \pm 0.03$ & $378 \pm 24$ & $86 \pm 5$ & $2.85 \pm 0.02$ & $226 \pm 20$ & $126 \pm 11$ \\
\hline Water/polycarb. & $0.0598 \pm 0.0005$ & - & - & $0.0916 \pm 0.0008$ & - & - \\
\hline Polycarb./air & $15.23 \pm 0.13$ & $387 \pm 5$ & $393 \pm 6$ & $12.98 \pm 0.11$ & $248 \pm 7$ & $523 \pm 15$ \\
\hline \multirow[t]{2}{*}{ Fatty/meaty ham } & $3.13 \pm 0.03$ & - & - & $2.93 \pm 0.02$ & - & - \\
\hline & \multicolumn{3}{|l|}{$\mathrm{TV}$} & \multicolumn{3}{|l|}{$\mathrm{RL}$} \\
\hline $\begin{array}{l}\text { Material } \\
\text { combination }\end{array}$ & $\begin{array}{l}\mathrm{CNR} / D^{1 / 2} \\
\left(\mathrm{mGy}^{-1 / 2}\right)\end{array}$ & $\begin{array}{l}\text { FWHM } \\
(\mu \mathrm{m})\end{array}$ & $\begin{array}{l}\mathrm{FOM} \times 10^{4} \\
\left(\mu \mathrm{m}^{-1} \mathrm{mGy}^{-1 / 2}\right)\end{array}$ & $\begin{array}{l}\mathrm{CNR} / D^{1 / 2} \\
\left(\mathrm{mGy}^{-1 / 2}\right)\end{array}$ & $\begin{array}{l}\text { FWHM } \\
(\mu \mathrm{m})\end{array}$ & $\begin{array}{l}\mathrm{FOM} \times 10^{4} \\
\left(\mu \mathrm{m}^{-1} \mathrm{mGy}^{-1 / 2}\right)\end{array}$ \\
\hline Glycerol/polycarb. & $2.43 \pm 0.02$ & $285 \pm 54$ & $\mathbf{8 5} \pm 16$ & $\mathbf{2 . 4 9} \pm 0.02$ & $935 \pm 51$ & $27 \pm 1$ \\
\hline $\mathrm{CaCl}_{2} /$ polycarb. & $\mathbf{8 . 3 5} \pm 0.07$ & $624 \pm 20$ & $\mathbf{1 3 3} \pm 4$ & $7.52 \pm 0.06$ & $808 \pm 25$ & $93 \pm 3$ \\
\hline Ethanol/polycarb. & $0.678 \pm 0.006$ & - & - & $\mathbf{0 . 7 1 7} \pm 0.006$ & - & - \\
\hline Paraffin/polycarb. & $4.27 \pm 0.04$ & $232 \pm 14$ & $\mathbf{1 8 4} \pm 11$ & $\mathbf{5 . 1 7} \pm 0.04$ & $613 \pm 18$ & $84 \pm 3$ \\
\hline Water/polycarb. & $0.151 \pm 0.001$ & - & - & $\mathbf{0 . 1 6 0} \pm 0.002$ & - & - \\
\hline Polycarb./air & $20.4 \pm 0.2$ & $203 \pm 4$ & $1006 \pm 22$ & $\mathbf{2 4 . 1} \pm 0.2$ & $624 \pm 8$ & $387 \pm 6$ \\
\hline Fatty/meaty ham & $4.76 \pm 0.04$ & - & - & $\mathbf{5 . 2 3} \pm 0.04$ & - & - \\
\hline
\end{tabular}

$\dagger \mathrm{FOM}=\mathrm{CNR} / D^{1 / 2} / \mathrm{FWHM}$.

spatial resolution and boundary sharpness, as quantified by the quality index $Q_{S}$ and the FOM, the TV-based CT reconstruction algorithm is advantageous while the RL algorithm is the second best, in terms of the quality index (which does not take into account the boundary sharpness) and is the worst in terms of the FOM which incorporates the boundary sharpness. In fact, this method was found to be the worst in terms of the degradation of the boundary sharpness.

The four iterative CT reconstruction algorithms compared in this section were used with the parameters provided in the

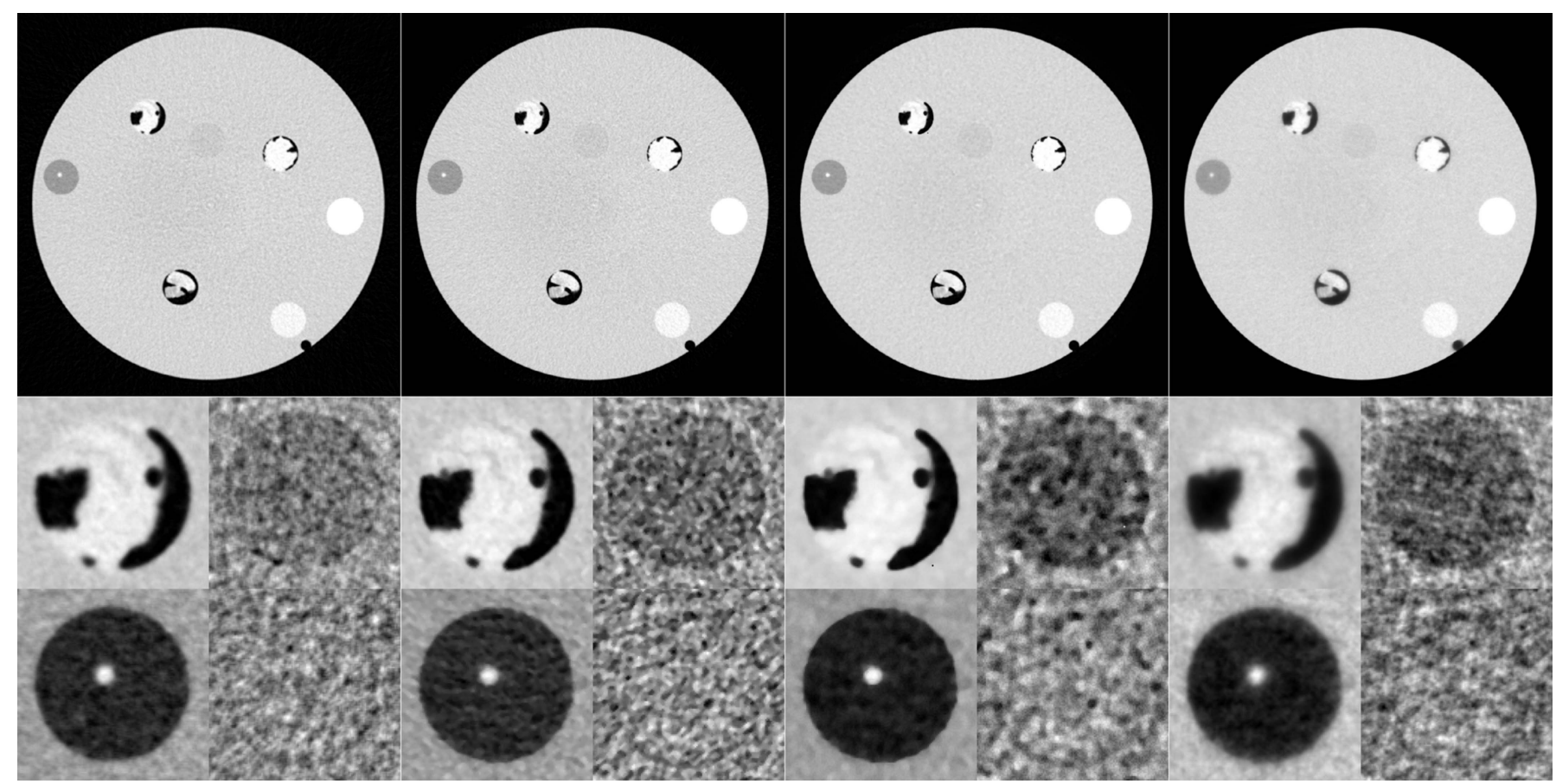

Figure 8

CT slice and its enlarged fragments reconstructed, using four iterative algorithms, from low-photon-statistics $\left(340\right.$ projections over $180^{\circ}$, for EST, 361 projections for the rest) phase-retrieved (TIE-HOM with $\gamma=1000)$ projection data set $\left(E=38 \mathrm{keV}, R_{2}=5 \mathrm{~m}\right.$ ). From left to right: EST reconstruction, iFBP, TV and RL. 
headnote to Table 13. For the EST algorithm, we followed the recommendation provided by the authors of the algorithm to use a few iterations of the gradient algorithm (we used five iterations). The iterative FBP algorithm was found to be sensitive to the noise-to-signal ratio (NSR) parameter. This value was estimated using the object-free fragments of the experimental sinograms. The same NSR value was also used in the TV and RL algorithms. In the case of the TV-based algorithm, we found that its convergence was typically observed after 10-20 iterations and the performance of the algorithm was strongly affected by the regularization parameter $\beta$ which set the relative weight of the total variation to the goodness-of-fit term in the cost function. With larger values of $\beta$, one obtains $\mathrm{CT}$ slices with less noise but more smeared details of the object. In the case of the RL algorithm, the number of iterations plays the role of a regularization parameter: by increasing the number of iterations one achieves a better match between the original and calculated sinograms, thus improving the resolution in the reconstructed slices, but amplifying noise. The regularization parameters in the TV and RL algorithms were chosen by visual inspection of the reconstructed slices and thus are very subjective. A more rigorous regularization approach for these two algorithms could be based on finding such regularization parameters that maximize the quality index $Q_{S}$ or the FOM. This tuning procedure has not been implemented in this paper and will be the subject of our future research.

\section{Conclusions}

The main outcome of this study is the demonstration of the possibility of producing high-quality X-ray in-line phasecontrast CT images with a conventional flat-panel detector having relatively large $(\sim 100 \mathrm{~mm})$ pixels. A key feature of this method is the use of long (up to $5 \mathrm{~m}$, in this study) distances between the sample and the detector, which does not induce penumbral blurring of the image as long as the incident X-ray beam has sufficiently high spatial coherence, as available at synchrotron beamlines and with microfocus laboratory X-ray sources. At the same time, the long effective propagation distances can lead to sufficient 'amplification' of in-line phase contrast to the extent that it becomes possible to detect and utilize it in spite of the relatively low spatial resolution (broad point-spread function) of the imaging system. Importantly, detectors with large pixels can typically afford a thicker and hence more efficient X-ray scintillator layer, thus resulting in higher quantum efficiency. This in turn allows one to solve the two critical issues which so far have prevented practical applications of in-line phase-contrast CT imaging for medical diagnostic purposes, namely that of long exposure times and large radiation doses (which were primarily due to the use of high-resolution detectors). In this work, we have analysed the effects of key imaging setup parameters, such as the X-ray energy, the sample-to-detector distance, the photon fluence (X-ray dose), as well as image processing steps, including the phase retrieval and advanced CT reconstruction algorithms, on the objective quality characteristics of the resultant images.
We have demonstrated that an optimal combination of the above factors can allow one to produce high-quality in-line phase-contrast CT images of soft-tissue samples at radiologically acceptable doses.

\section{Acknowledgements}

We are grateful to Dr Nicola Sodini of Elettra-Sincrotrone Trieste for manufacturing the plastic phantom used in this study. We are also grateful to Ms Clare Scott and Dr Anton Maksimenko of the Australian Synchrotron for the assistance in preparation of the samples and for help in conducting the experiment, respectively. We are also grateful to Dr Jessica Lye of ARPANSA/RMIT for calculating the electron-loss correction factors for an ion chamber used in the experiment. KMP acknowledges financial support from the University of New England. MJK acknowledges funding from the Australian Research Council (ARC) and is an ARC Australian Research Fellow (DP110101941). This research was undertaken on the Imaging and Medical beamline at the Australian Synchrotron, Victoria, Australia.

\section{References}

Beltran, M. A., Paganin, D. M., Siu, K. K., Fouras, A., Hooper, S. B., Reser, D. H. \& Kitchen, M. J. (2011). Phys. Med. Biol. 56, $7353-$ 7369.

Bravin, A., Coan, P. \& Suortti, P. (2013). Phys. Med. Biol. 58, R1-R35.

Ciatto, S., Houssami, N., Bernardi, D., Caumo, F., Pellegrini, M., Brunelli, S., Tuttobene, P., Bricolo, P., Fantò, C., Valentini, M., Montemezzi, S. \& Macaskill, P. (2013). Lancet Oncol. 14, 583-589.

Dance, D. R. (1990). Phys. Med. Biol. 35, 1211-1220.

Diemoz, P. C., Bravin, A., Langer, M. \& Coan, P. (2012). Opt. Express, 20, 27670-27690.

Gureyev, T. E., Mayo, S. C., Nesterets, Y. I., Mohammadi, S., Lockie, D., Menk, R. H., Arfelli, F., Pavlov, K. M., Kitchen, M. J., Zanconati, F., Dullin, C. \& Tromba, G. (2014a). J. Phys. D, 47, 365401.

Gureyev, T., Mohammadi, S., Nesterets, Y., Dullin, C. \& Tromba, G. (2013). J. Appl. Phys. 114, 144906.

Gureyev, T. E., Nesterets, Y. I., de Hoog, F., Schmalz, G., Mayo, S. C., Mohammadi, S. \& Tromba, G. (2014b). Opt. Express, 22, 90879094.

Gureyev, T. E., Nesterets, Y., Ternovski, D., Thompson, D., Wilkins, S. W., Stevenson, A. W., Sakellariou, A. \& Taylor, J. A. (2011). Proc. SPIE, 8141, 1-14.

Gureyev, T. E., Stevenson, A. W., Nesterets, Y. I. \& Wilkins, S. W. (2004). Opt. Commun. 240, 81-88.

Hammerstein, R. G., Miller, D. W., White, D. R., Masterson, E. M., Woodard, H. Q. \& Laughlin, J. S. (1979). Radiology, 130, 485-491. Johns, P. C. \& Yaffe, M. J. (1985). Med. Phys. 12, 289-296.

Lucy, L. B. (1974). Astron. J. 79, 745-754.

Malliori, A., Bliznakova, K., Speller, R. D., Horrocks, J. A., Rigon, L., Tromba, G. \& Pallikarakis, N. (2012). Med. Phys. 39, 5621-5634.

Miao, J., Förster, F. \& Levi, O. (2005). Phys. Rev. B, 72, 052103.

Myers, G. R., Thomas, C. D. L., Paganin, D. M., Gureyev, T. E. \& Clement, J. G. (2010). Appl. Phys. Lett. 96, 021105.

NCRP (2004). A Guide to Mammography and Other Breast Imaging Procedures. NCRP Report No. 149. Bethesda, MD: National Council on Radiation Protection and Measurements.

Nesterets, Y. I. \& Gureyev, T. E. (2009). Proceedings of the 18th World IMACS Congress and MODSIM09 International Congress on Modelling and Simulation, edited by R. S. Anderssen, R. D. Braddock \& L. T. H. Newham, pp. 1045-1051.

Nesterets, Y. I. \& Gureyev, T. E. (2014). J. Phys. D, 47, 105402. 
Olivo, A., Gkoumas, S., Endrizzi, M., Hagen, C. K., Szafraniec, M. B., Diemoz, P. C., Munro, P. R., Ignatyev, K., Johnson, B., Horrocks, J. A., Vinnicombe, S. J., Jones, J. L. \& Speller, R. D. (2013). Med. Phys. 40, 090701.

Pacilè, S., Brun, F., Dullin, C., Nesterets, Y. I., Dreossi, D., Mohammadi, S., Tonutti, M., Stacul, F., Lockie, D., Zanconati, F., Accardo, A., Tromba, G. \& Gureyev, T. E. (2015). Biomed. Opt. Express, 6, 3099.

Paganin, D., Mayo, S. C., Gureyev, T. E., Miller, P. R. \& Wilkins, S. W. (2002). J. Microsc. 206, 33-40.

Pisano, E. D., Gatsonis, C., Hendrick, E., Yaffe, M., Baum, J. K., Acharyya, S., Conant, E. F., Fajardo, L. L., Bassett, L., D’Orsi, C., Jong, R. \& Rebner, M. (2005). N. Engl. J. Med. 353, 1773-1783.

Richardson, W. H. (1972). J. Opt. Soc. Am. 62, 55-59.

Rose, A. (1948). J. Opt. Soc. Am. 38, 196-208.

Sijbers, J. \& Postnov, A. (2004). Phys. Med. Biol. 49, N247-N253.
Stevenson, A. W., Mayo, S. C., Häusermann, D., Maksimenko, A., Garrett, R. F., Hall, C. J., Wilkins, S. W., Lewis, R. A. \& Myers, D. E. (2010). J. Synchrotron Rad. 17, 75-80.

Teague, M. R. (1983). J. Opt. Soc. Am. 73, 1434-1441.

Thompson, D., Nesterets, Y. I., Gureyev, T. E., Sakellariou, A., Khassapov, A. \& Taylor, J. A. (2011). Proceedings of MODSIM2011 - 19th International Congress on Modelling and Simulation, edited by F. Chan, D. Marinova and R. S. Anderssen, Perth, Australia, 12-16 December 2011, pp. 620-626.

X-TRACT (2015). X-TRACT, http://www.ts-imaging.net/Services/ AppInfo/X-TRACT.aspx.

Zhao, Y. Z., Brun, E., Coan, P., Huang, Z. F., Sztrókay, A., Diemoz, P. C., Liebhardt, S., Mittone, A., Gasilov, S., Miao, J. W. \& Bravin, A. (2012). Proc. Natl Acad. Sci. USA, 109, 18290-18294.

Zysk, A. M., Brankov, J. G., Wernick, M. N. \& Anastasio, M. A. (2012). Med. Phys. 39, 906-911. 